ARTICLE

\title{
Developmental divergence of sensory stimulus representation in cortical interneurons
}

\author{
Rahel Kastli ${ }^{1,2,5}$, Rasmus Vighagen 1,2,5, Alexander van der Bourg ${ }^{1,2,5}$, Ali Özgür Argunsah (10) 1,2,5, Asim Iqbal (1) 1,2, \\ Fabian F. Voigt (1D) ${ }^{2,3}$, Daniel Kirschenbaum ${ }^{2,4}$, Adriano Aguzzi (1D ${ }^{2,4}$, Fritjof Helmchen (1) ${ }^{2,3}$ \& \\ Theofanis Karayannis (1) 1,2凶
}

\begin{abstract}
Vasocative-intestinal-peptide ( $\left.\mathrm{VIP}+{ }^{+}\right)$and somatostatin $\left(\mathrm{SST}^{+}\right)$interneurons are involved in modulating barrel cortex activity and perception during active whisking. Here we identify a developmental transition point of structural and functional rearrangements onto these interneurons around the start of active sensation at P14. Using in vivo two-photon $\mathrm{Ca}^{2+}$ imaging, we find that before P14, both interneuron types respond stronger to a multi-whisker stimulus, whereas after P14 their responses diverge, with $\mathrm{VIP}+$ cells losing their multi-whisker preference and $\mathrm{SST}^{+}$neurons enhancing theirs. Additionally, we find that $\mathrm{Ca}^{2+}$ signaling dynamics increase in precision as the cells and network mature. Rabies virus tracings followed by tissue clearing, as well as photostimulation-coupled electrophysiology reveal that $\mathrm{SST}^{+}$cells receive higher cross-barrel inputs compared to $\mathrm{VIP}^{+}$neurons at both time points. In addition, whereas prior to P14 both cell types receive direct input from the sensory thalamus, after $\mathrm{P} 14 \mathrm{VIP}+$ cells show reduced inputs and $\mathrm{SST}^{+}$cells largely shift to motorrelated thalamic nuclei.
\end{abstract}

\footnotetext{
${ }^{1}$ Laboratory of Neural Circuit Assembly, Brain Research Institute, University of Zurich, Winterthurerstrasse 190, CH-8057 Zurich, Switzerland.

${ }^{2}$ Neuroscience Center Zurich, Winterthurerstrasse 190, CH-8057 Zurich, Switzerland. ${ }^{3}$ Laboratory of Neural Circuit Dynamics, Brain Research Institute, University of Zurich Winterthurerstrasse 190, CH-8057 Zurich, Switzerland. ${ }^{4}$ Institute of Neuropathology, University Hospital Zurich, Schmelzbergstrasse 12 , CH-8091 Zurich, Switzerland. ${ }^{5}$ These authors contributed equally: Rahel Kastli, Rasmus Vighagen, Alexander van der Bourg, Ali Özgür Argunsah.

凶email: karayannis@hifo.uzh.ch
} 
$\mathrm{P}$ ostnatal development and maturation of neuronal circuits responsible for sensory processing is fundamental for accurate representation of the environment as animals transition into actively interacting with the external world ${ }^{1}$. Unravelling the mechanisms of neocortical development is key for understanding the emergence of purposeful and goal-directed motor actions informed by sensory cues. It is therefore of great importance to study the alterations that sensory cortical networks undergo in responding to diverse sensory stimuli upon developmental behavioural transitions.

The whisker primary somatosensory cortex (wS1) of rodents is a well-suited model to study sensory processing throughout development due to the somatotopic way in which the information is transmitted from the whiskers to the cortex, as well as its role in spatial navigation ${ }^{2,3}$. Although the whiskers of mice are already present at the time of birth, during the first postnatal weeks the whisker pad only exhibits spontaneous muscle twitches, which coincide with activity in $\mathrm{wS}^{4,5}$. Around postnatal day 14 (P14), mice start displaying bilateral rhythmic movements of the whiskers ("active whisking"), enabling them to explore their environment and extract more detailed information from their surrounding ${ }^{6-8}$.

Although the wS1 has been a subject of research for many decades, it is only recently that the role of inhibition in sensory processing in the adult cortex has started to be explored. Several studies showed that layer 2/3 (L2/3) vasoactive intestinal peptideexpressing $\left(\mathrm{VIP}^{+}\right)$interneurons (INs) in the wS1 can inhibit the inhibitory somatostatin-expressing ( $\mathrm{SST}^{+}$) INs, thus leading to a net excitation of pyramidal cells (disinhibition) ${ }^{9-11}$. The same disinhibitory connectivity motif has also been described in other cortical areas, suggesting a general mechanism by which corticocortical loops can influence cortical processing ${ }^{12-16}$. In the $\mathrm{wS} 1$, this $\mathrm{VIP}^{+}{ }_{-} \mathrm{SST}^{+}$disinhibitory loop can be recruited in a topdown fashion by the whisker primary motor cortex (wM1), which directly innervates the $\mathrm{wS}_{1} \mathrm{VIP}^{+}$INs in $\mathrm{L} 2 / 3^{9}$. In addition, it has been shown that $\mathrm{VIP}^{+}$INs can be activated by bottom-up thalamic inputs, potentially also engaging in disinhibition through $\mathrm{SST}^{+}$cells ${ }^{17-19}$. Nevertheless, a recent study reports opposite results, with $\mathrm{SST}^{+}$cells being strongly activated by a whiskerdriven sensory stimulus, compared to $\mathrm{VIP}^{+}$cells which are found to be silenced ${ }^{20}$. We hypothesized that the main reason behind this discrepancy between the aforementioned results is the stimulus paradigm used. Researchers either stimulated one single whisker ${ }^{11}$ or multiple whiskers at the same time ${ }^{20}$, two paradigms that would engage the adult barrel cortex in very distinct ways. We further hypothesized that these INs would be differently engaged by these stimulation paradigms prior to P14, when topdown modulation is absent and discrimination of fine features through the somatosensory system is probably not yet developed. When we tested these hypotheses, we indeed found that the strength of activation of $\mathrm{VIP}^{+}$and $\mathrm{SST}^{+}$INs depends on the nature of the presented stimulus. We find that compared to the single-whisker deflection, the multi-whisker stimulation leads to a higher activation of both cell types before P14, a phenomenon only maintained in $\mathrm{SST}^{+}$after the onset of whisking. Intriguingly, these response alterations are accompanied by a significant rearrangement of thalamic connections onto both $\mathrm{VIP}^{+}$ and $\mathrm{SST}^{+}$INs in the same time window, later than any other thalamo-cortical connectivity restructuring reported to date.

\section{Results}

Divergent sensory stimulus responses in superficial cortical $\mathrm{VIP}^{+}$and SST $^{+}$interneurons during development. To assess how wS1 $\mathrm{VIP}^{+}$and $\mathrm{SST}^{+}$INs in $\mathrm{L} 2 / 3$ respond to whisker stimuli across development we performed acute in vivo two-photon calcium $\left(\mathrm{Ca}^{2+}\right)$ imaging under light-anesthesia at two developmental time points; prior to the onset of active whisking (age: P8-12) and after the beginning of active whisking (age: P21-41, denoted as $\mathrm{P} 21+$ ). To visualize $\mathrm{VIP}^{+}$and $\mathrm{SST}^{+}$IN activity, we used animals expressing tdTomato in either $\mathrm{VIP}^{+}$or $\mathrm{SST}^{+}$INs (VIPCre-Ai14 and SSTCre-Ai14 lines, respectively) and injected the membrane-permeable AM-ester form of the $\mathrm{Ca}^{+}$indicator OGB-1 into wS1. Two-photon $\mathrm{Ca}^{2+}$ imaging was performed during spontaneous activity and upon stimulation of either the principal C2 whisker one time alone (single-whisker stimulation) or both the principal whisker and the majority of the macro vibrissae once together (multi-whisker stimulation) (Fig. 1b) ${ }^{21}$.

We first tested whether $\mathrm{VIP}^{+}$and $\mathrm{SST}^{+}$INs are functionally integrated before P14 by examining their spontaneous activity. We observed prominent $\mathrm{Ca}^{2+}$ transients in both IN types that correlated with the activity of the surrounding cells (Figs. 1c and S1a), indicating that they receive input capable of driving action potentials before the onset of active whisking. Previous studies have shown that cortical pyramidal neurons and superficial $5 \mathrm{HT} \mathrm{aR}^{+}$INs display highly correlated spontaneous activity within their population in early postnatal development and then undergo a de-correlation upon maturation of the circuit ${ }^{21-24}$. To test if the same is true for $\mathrm{VIP}^{+}$and $\mathrm{SST}^{+}$INs, we compared the correlations of spontaneous activity within each IN population before and after P14 and found a significant drop of co-activation with time (Fig. 1e). Interestingly, at $\mathrm{P} 21+$, spontaneous activity of $\mathrm{SST}^{+}$cells is more correlated than that of $\mathrm{VIP}^{+}$cells, a phenomenon that has also been described in the visual cortex of adult mice $(p<0.01 \text {, comparison not shown in the figure })^{25}$. In addition, when we compared the average correlation between $\mathrm{SST}^{+}$and $\mathrm{VIP}^{+}$INs and between the IN subtypes and their surrounding non-labeled cells, most likely excitatory neurons, we found a similar decorrelation (Fig. S1a).

Having established that $\mathrm{VIP}^{+}$and $\mathrm{SST}^{+}$INs are embedded in the developing circuit prior to P14, we next tested if they respond to whisker stimulation and if yes, how this would potentially change across development (Fig. 1b). Since in a natural environment, both single and multiple whiskers can be deflected, we used a single- and a multi-whisker stimulus in both age groups, with the latter being even more relevant for neonatal mice, whose whiskers are physically closer together ${ }^{5}$.

As the response to a single-whisker stimulus in adult L2/3 $\mathrm{VIP}^{+}$and $\mathrm{SST}^{+}$INs has been described before by cell-attached electrophysiological recordings ${ }^{17}$, we first compared the activity of the two cell types in response to this stimulation paradigm. We found that at $\mathrm{P} 21+$ the peak $\Delta F / F$ of the average $\mathrm{Ca}^{+}$response in $\mathrm{SST}^{+}$INs was lower compared to that of $\mathrm{VIP}^{+}$INs, matching the published data ${ }^{17}$ (Fig. S1b). Nevertheless, when looking at the integral of $\Delta F / F$ average response (over $8 \mathrm{~s}$ following whisker stimulation) there was no significant difference between the two cell types (Fig. S1b), and the same was true before P14 (Fig. S1c).

We then assessed how IN responses to multi- compared to single-whisker stimulation may change during development by comparing the $\Delta F / F$ integral as an overall measure of direct or indirect activation of the cells by the sensory stimuli. The analysis showed that before the onset of active whisking (P8-12), both $\mathrm{VIP}^{+}$and $\mathrm{SST}^{+}$INs responded significantly stronger to multicompared to single-whisker stimulation (Fig. 1d, f). For the $\mathrm{SST}^{+}$ INs this continued to be the case and even enhanced after the onset of active whisking $(\mathrm{P} 21+)$, while for the $\mathrm{VIP}^{+}$INs the $\Delta F / F$ integral became similar between multi- versus single-whisker stimulation (Fig. 1f).

Since the $\mathrm{Ca}^{2+}$ transients recorded at $\mathrm{P} 8-12$ had a clear late component and lower peak amplitude than the mature cells, we sought to better understand how they relate to action potentials 
a

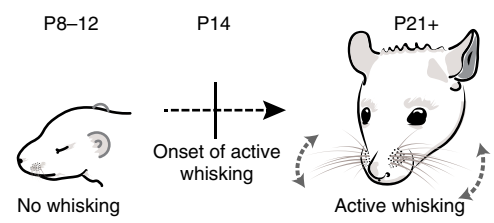

C

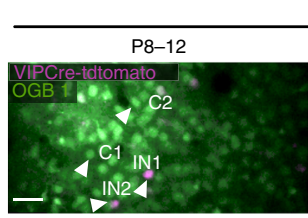

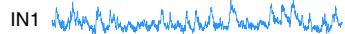

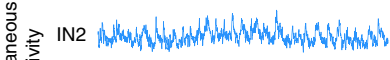
空

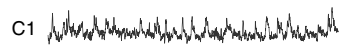

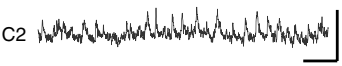

VIP

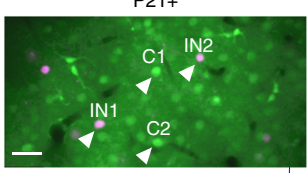

(1)

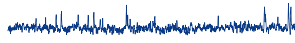

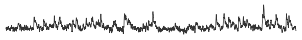

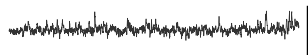

d

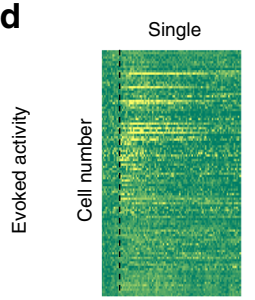

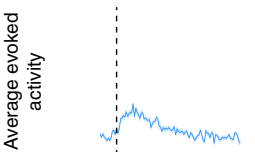
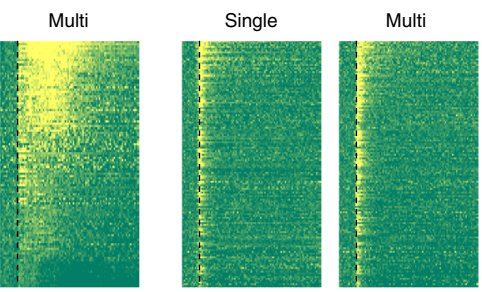

e
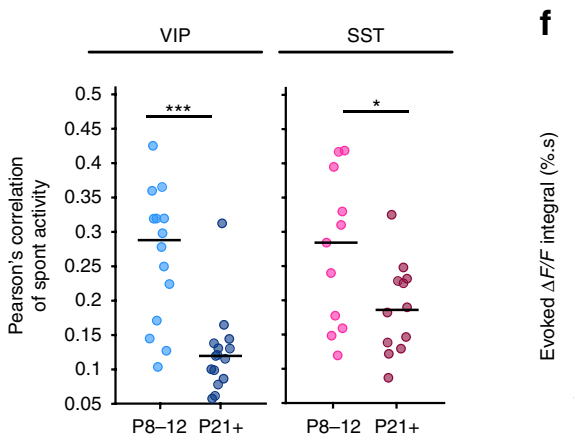

f b

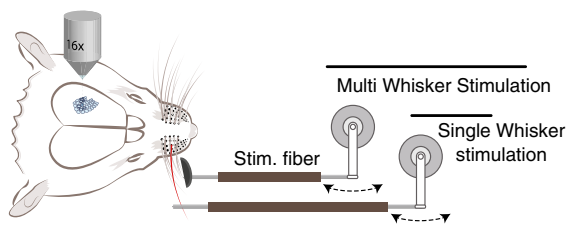

SST

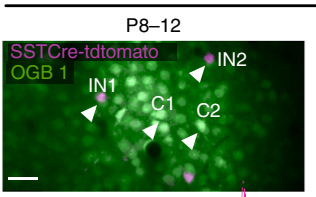

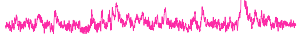

and

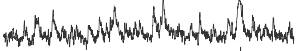

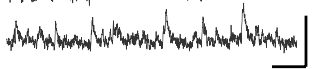

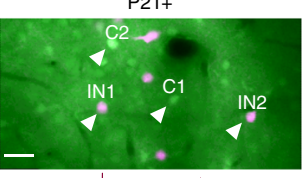

.

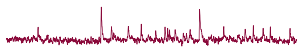

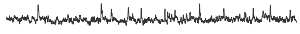
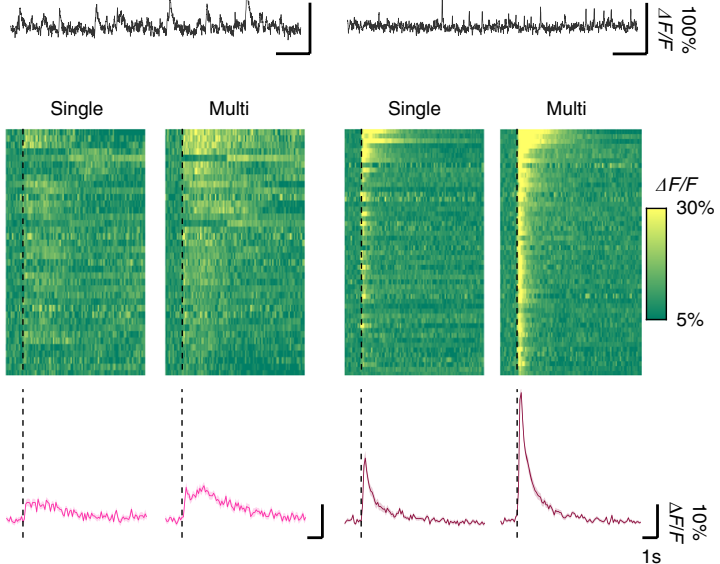

$\int_{1 \mathrm{~s}} \min _{0}^{\circ}$
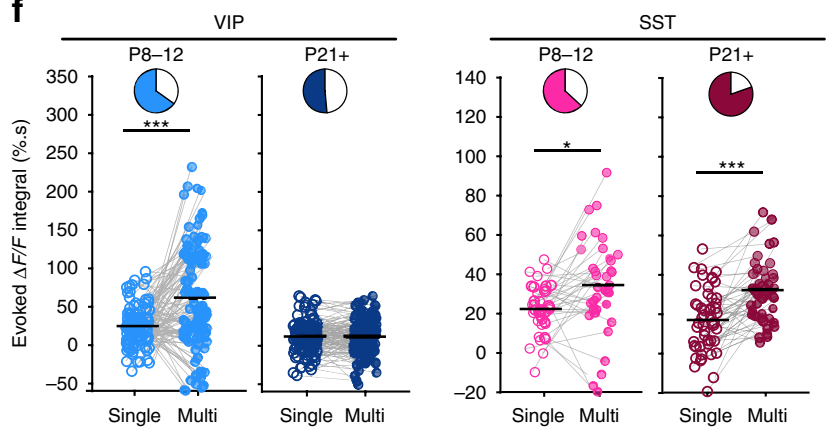

g

VIP

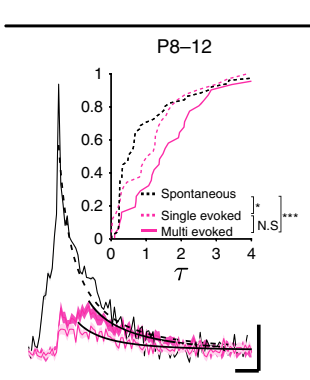

SST
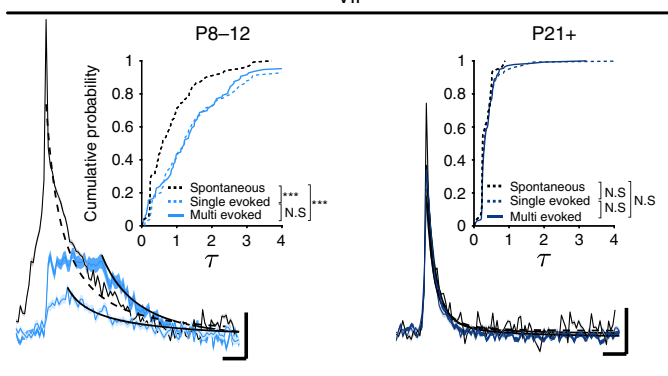

across development. We therefore filled SST ${ }^{+}$INs with OGB-1 in vitro and evoked a set number of action potentials (APs), while recording the $\mathrm{Ca}^{2+}$ transients in the cell bodies using two-photon microscopy. We found, that when comparing the $\Delta F / F$ responses between the two age groups, there was no significant difference, indicating that the integral of the $\mathrm{Ca}^{2+}$ responses could provide a good estimate for the functional activation of the cell types across development (Fig. S1d). Nonetheless, it is worth noting that at both ages the time constant of the decay (tau) increases with the number of APs elicited, with the $\mathrm{Ca}^{2+}$ response to $10 \mathrm{APs}$ 
Fig. 1 Divergent sensory stimulus responses in superficial cortical VIP ${ }^{+}$and $\mathbf{S S T}^{+}$interneurons during development. a Acute in vivo two-photon $\mathrm{Ca}^{2+}$ imaging was performed before (P8-12) and after (P21+) the start of active whisking. b Schematic represenation of the whisker stimulation protocol. c Top : average intensity projection of $\mathrm{Ca}^{2+}$ imaging regions after bulk loading of OGB-1 (green). Interneurons are labeled with tdTomato using reporter mouse lines; scale bar $35 \mu \mathrm{m}$. Bottom: representative examples of raw $\Delta F / F$ traces of spontaneous activity of two interneurons (IN1/2) and two surrounding cells $(C 1 / 2)$. ( $N=3$ animals per group,VIP P8-12:109 cells, SST P8-12: 38 cells, VIP P21+: 138 cells, SST P21+: 51 cells). d Top: $\Delta F / F$ signal over time of all recorded VIP and SST cells after whisker stimulation. $\mathrm{Ca}^{2+}$ responses are baseline corrected and aligned to whisker stimulus onset (dashed line). Cells are sorted by their cummulative activity following multi-whisker stimulation. Same number of cells are included as in $\mathbf{c}$. Bottom: Average $\Delta F / F$ responses with SEM. e Pearson's correlations of spontaneous neuronal activity within each interneuron type before- and after P14. Same number of cells are included as in c, but the data are plotted by imaging spot. Statistics: two-sided Mann-Whitney $U$ test, $p(V I P)=6.298 \times 10^{-5}, p(S S T)=0.047$. f Distribution of average values of the evoked $\Delta F / F$ integral after single and multi whisker stimulation for all cells analyzed. Pie charts show the fraction of cells that increase (coloured) or decrease (white) in activity by multi vs. single whisker stimulation. Same number of cells as in c. Statistics: two-sided paired Wilcoxonsigned-rank test, $p($ VIP P8-12 $)=1.368 \times 10^{-5}, p($ SST P8-12 $)=0.011, p($ SST P21+ $)=5.576 \times 10^{-7}$. g Average $\Delta F / F$ traces with SEM of evoked and spontaneous events. Fitted curve of decay time constant is plotted in black (continous line: evoked, dotted line: spontaneous). Insets show the cummulative distribution of the decay tau. Statistics: two-sample Kolmogorov-Smirnov test; VIP P8-12: $p$ (spont vs. single) $=1.257 \times 10^{-14}, p($ spont vs.multi) $=2.611 \times$ $10^{-5}$; SST P8-12: $p$ (spont vs. single $)=0.034, p$ (spont vs. multi $)=2.615 \times 10^{-4}$. Source data are providedas a Source Data file.

returning to baseline two-times slower than the response to $1 \mathrm{AP}$ (Fig. S1d). This non-linearity could either be caused by saturation of $\mathrm{Ca}^{2+}$ extrusion mechanisms when the cell is repeatedly activated or due to the diffusion of $\mathrm{Ca}^{2+}$ buffer between the cell soma and the patching pipet during the experiment. Furthermore, even though the prolongation in decay tau after multiple APs is about two-fold at both ages, at P8-12 the $\Delta F / F$ integral increases much more than that (Fig. S1d), likely due to the immature state of the neurons.

To further investigate developmental changes in $\mathrm{Ca}^{2+}$ signaling we analyzed the decay of spontaneous activity at P8-12 and P21+ and found that both in $\mathrm{VIP}^{+}$and $\mathrm{SST}^{+} \mathrm{INs}^{2} \mathrm{Ca}^{2+}$ dynamics sharpen as the animal matures, with spontaneous transients dissipating faster at P21+ than at P8-12 (Fig. S1e). We then compared the decay tau of spontaneous and evoked activity in $\mathrm{VIP}^{+}$and $\mathrm{SST}^{+}$at the two different time points. Our results show that at $\mathrm{P} 21+$ the decay of $\mathrm{Ca}^{2+}$ signal is very similar, regardless of the cell type and of how the activity was elicited (spontaneous, single whisker, multi whisker) (Fig. 1g). At P8-12 on the other hand, the decay of spontaneous activity is much faster than that of evoked activity (Fig. 1g). Furthermore, spontaneous events show a larger amplitude and shorter duration than evoked ones (Fig. 1g). The data suggest that the pronounced late component of the evoked $\mathrm{Ca}^{2+}$ signal at P8-12 is not due to immature $\mathrm{Ca}^{2+}$ buffering capacity, but rather due to the way the network activates the cells. Finally, to address if the sensory-evoked $\mathrm{Ca}^{2+}$ responses we observed for the cell types and stimuli across development carry information that can make them discriminatory, we trained three different decoders to quantify differences in response profiles. Each decoder was trained on a subset of $\mathrm{Ca}^{2+}$ responses, evoked through either multi- or single-whisker stimulation, for each IN type and age. All of the classifiers showed above chance level values with VIPs displaying a reduction with age, whereas the SSTs an increase. This finding lends further support for the developmental divergence of these two cell type's activation and function (Fig. S1f).

Superficial VIP $^{+}$and SST $^{+}$interneurons show distinct barrelfield afferent connectivity motifs. Increased responsiveness to multi- over single-whisker stimulation at both ages could be due to the cortico-cortical inputs originating from surrounding barrel columns, which would provide convergent excitation originating from multiple whiskers. We therefore aimed at investigating the presynaptic inputs onto $\mathrm{L}_{2} / 3 \mathrm{VIP}^{+}$and $\mathrm{SST}^{+}$INs at the two developmental time points, using a monosynaptic rabies virus approach $^{26}$, which allowed us to assess both local and long-range connectivity.

By utilizing compound mouse genetics (VIPCre-HTB or SSTCre-HTB), in combination with the pseudo-typed rabies virus, direct presynaptic partners of $\mathrm{L}_{2} / 3 \mathrm{VIP}^{+}$and $\mathrm{SST}^{+} \mathrm{INs}$ were labeled with mCherry. The primary infected cells (starter cells) could be identified by double labeling of mCherry and eGFP (provided by the HTB line). The viral injections were done at either P5 or P15 and after 7 days the brains were collected (at P12 or P22 respectively). A CLARITY-based tissue clearing protocol was performed on the brains and they were subsequently imaged in their entirety using a custom-built light-sheet microscope (mesoSPIM) (Fig. 2a) ${ }^{27}$. The auto-fluorescence of the barrels allowed for the detection of the barrel field and enabled us to accurately localize our injection site within the wS1 (Figs. 2b-e and S2). A second batch of injected brains was processed using classical histology, followed by eGFP immunostaining and widefield imaging. Both the cleared whole brain and the histology dataset were used to quantify the number and laminar position of the starter and the presynaptic cells located around the wS1 injection site. This dual approach was performed to safeguard that no information was lost while clearing the brains. For the histology dataset, a subset of all cut sections was used to identify the cells manually, whereas in the cleared brains a deep-neural network was trained to detect and count cells ${ }^{28}$. For both methods, the results were highly comparable (Fig. S3).

The analysis showed that although there are starter cells in L26 at both time points we get robust primary infection in L2/3 for both $\mathrm{VIP}^{+}$and $\mathrm{SST}^{+}$cells (Fig. S3a, b). The distribution of the presynaptic cells across the layers mirrored that of the starter cells, with the $\mathrm{VIP}^{+}$INs receiving more inputs from the upper cortical layers while the $\mathrm{SST}^{+}$neurons more from the deeper layers (Fig. S3c, d).

To assess if $\mathrm{L}^{2 / 3} \mathrm{SST}^{+}$and $\mathrm{VIP}^{+}$INs receive a significant proportion of their inputs from neighboring barrels, the cleared $3 \mathrm{D}$ brain images were used to localize both starter and presynaptic cells within the barrel field (Figs. 2b-e and S2a, b). Only L2/3 presynaptic neurons located within wS1 were included in the analysis, since it is most likely that these inter-columnar inputs would originate from the upper layers ${ }^{29,30}$. As it is not possible to determine which presynaptic partner connects to which starter cell, a probabilistic approach was taken, where the distance between every starter to every presynaptic cell within $800 \mu \mathrm{m}$ distance was calculated and compared between the two cell types (Fig. S4). The analysis revealed that $\mathrm{SST}^{+}$, compared to $\mathrm{VIP}^{+}$INs, receive on average more distant inputs, both before and after P14 (Fig. 2f). To further validate the results and overcome the variability observed in the data we also calculated the cumulative distance distribution of randomly selected starter and presynaptic cells (Fig. S2c) (Online Methods). This anatomical data can help explain why the $\mathrm{SST}^{+}$cells are able to report multi- over single-whisker activation both before and after 
a

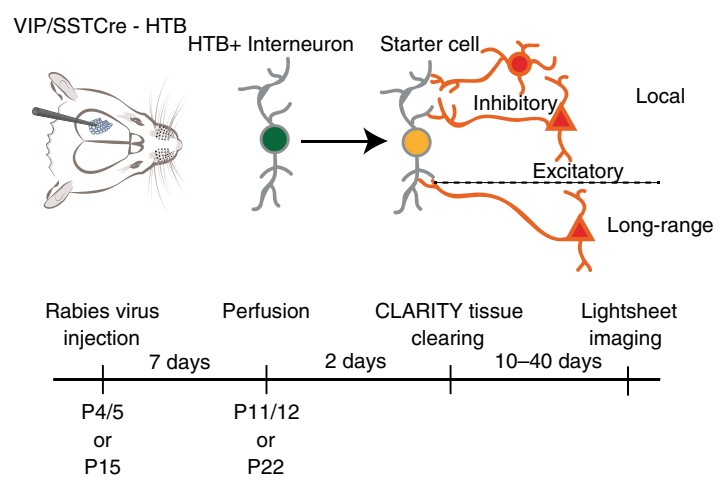

b
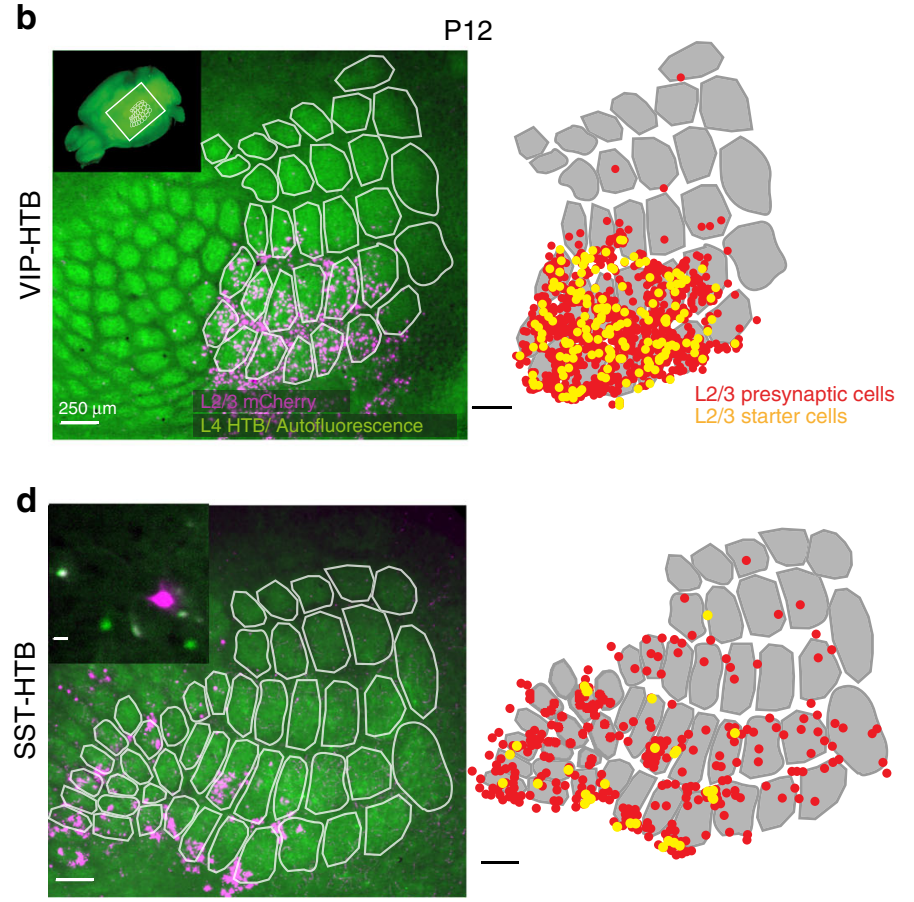

f

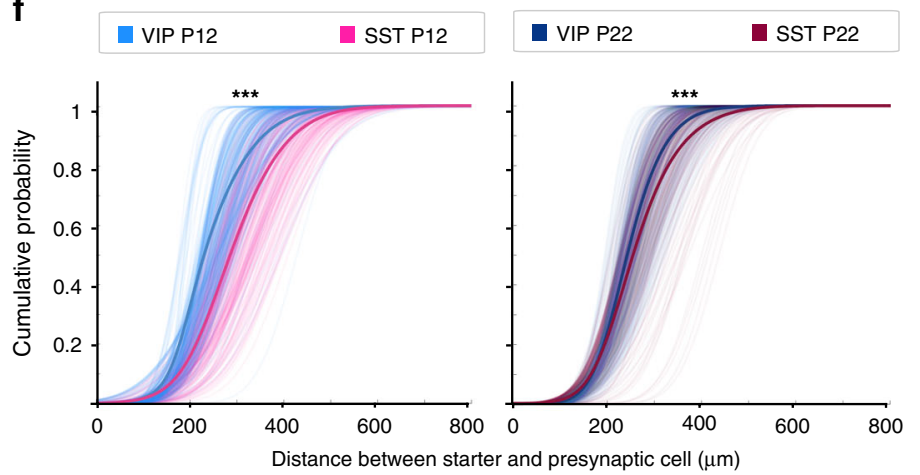

C

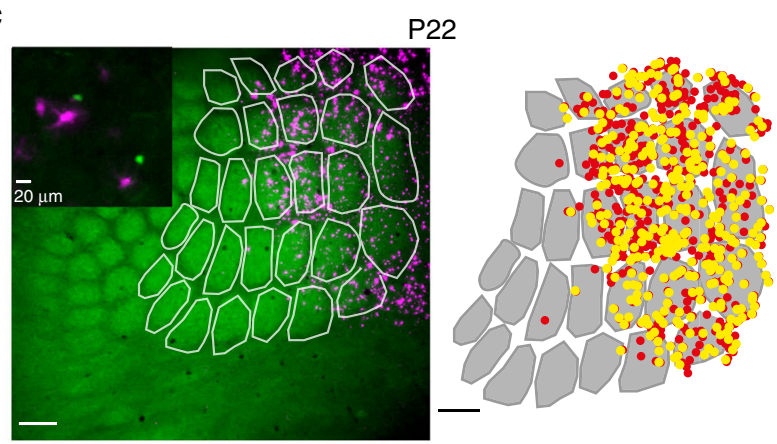

e

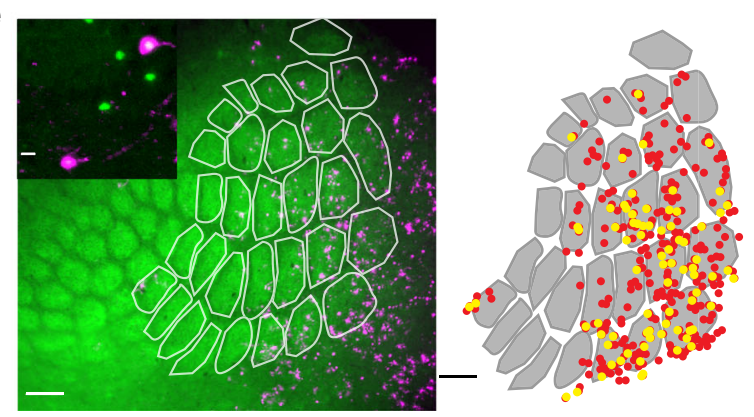

Fig. 2 Superficial VIP $^{+}$and SST ${ }^{+}$interneurons show distinct barrel-field afferent connectivity motifs. a Shematic representation of the experimental protocol. Rabies virus tracing was combined with tissue clearing and whole brain imaging. $\mathbf{b}$-e Left: overlay of maximum intensity projection of $L 2 / 3$ mCherry signal and median intensity projection of $L 4$ autofluorescence after re-slicing of whole brain images. Right: Tranformation applied before distance analysis. Segmented barrels overlayed with $L 2 / 3$ starter (yellow) and presynaptic (red) cells represented as dots. Inset in $\mathbf{b}$ indicates location of barrel field in the whole brain. Insets in c-e show close-ups of rabies labelled neurons. (VIP P12, N=4: 382 starter and 1943 presynaptic cells, SST P12, N=6: 102 starter and 581 presynaptic cells, VIP P22, N=5: 645 starter and 1561 presynaptic cells, SST P22, N=4: 110 starter and 504 presynaptic cells). f Cumulative distribution of Euclidian distance in 2D between the starter cells and all presynaptic cells in a $800-\mu \mathrm{m}$ radius around them. The faint lines in the background depict the distance distribution of each starter cell; the thicker lines depict the average. Same number of animals and cells as in b, c. Statistics: two-sample Kolmogorov-Smirnov test, $p(P 12)=8.410 \times 10^{-7}, p(P 22)=3.628 \times 10^{-6}$. Source data are provided as a Source data file.

P14. However, it cannot explain why the $\mathrm{VIP}^{+}$INs are able to differentiate between multi- and single-whisker stimulation before P14 and not after, unless there is a change in the functional connectivity.

Distinct functional connectivity motifs onto superficial VIP ${ }^{+}$ and $\mathrm{SST}^{+}$interneurons. After revealing the anatomical connections of the wS1 cortical inputs onto superficial $\mathrm{VIP}^{+}$and $\mathrm{SST}^{+}$INs, we assessed their functionality and strength. Specifically, we investigated if after $\mathrm{P} 14, \mathrm{SST}^{+}$INs receive stronger functional input from lateral sources compared to $\mathrm{VIP}^{+}$INs, while before P14 the two cell types display more functionally similar input.
In order to study the developmental trajectory of $\mathrm{VIP}^{+}$and $\mathrm{SST}^{+}$IN intrinsic properties we performed whole-cell current-

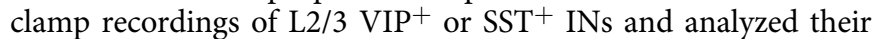
passive and active electrophysiological membrane properties (Figs. $3 b$ and S5a-e). As expected, we found evidence of cell maturation in both cell types, with the membrane resistance decreasing and the action potentials becoming faster (Fig. S5a, d, e). Nevertheless, the threshold for action potential generation dropped and action potential amplitude increased only in $\mathrm{VIP}^{+}$ INs, (Fig. S5b, c), suggesting that the $\mathrm{SST}^{+}$cells are closer to their mature state at P8-12.

In order to functionally analyze the distribution of neurons providing synaptic input to the two IN types, we performed glutamate uncaging. This was carried out using light 


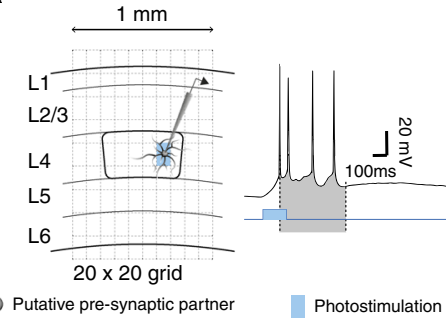

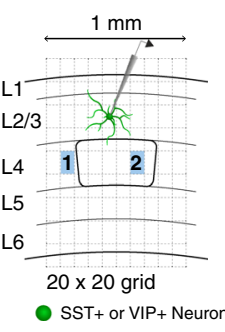

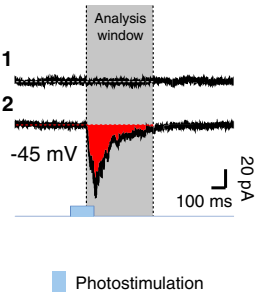

b

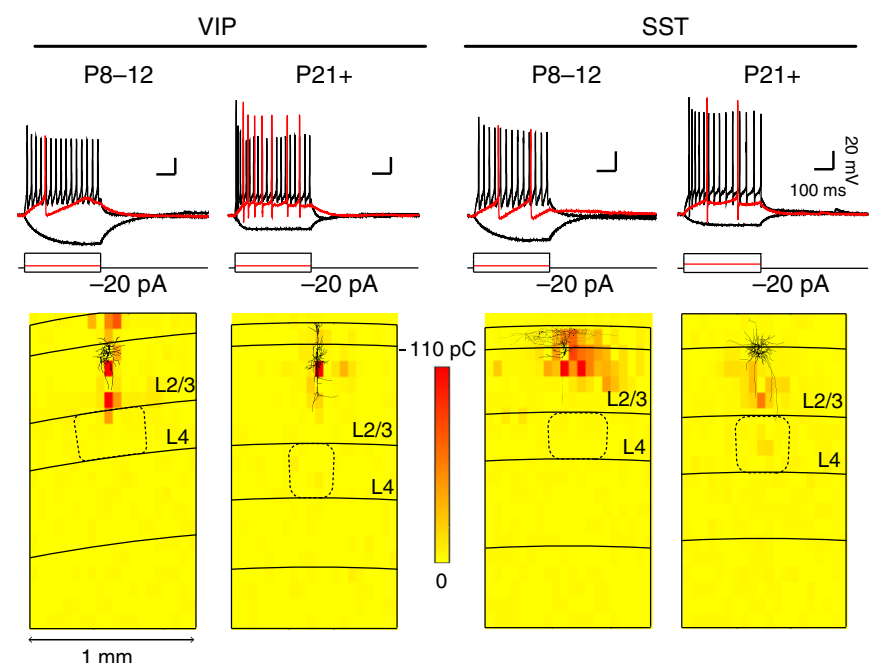

C

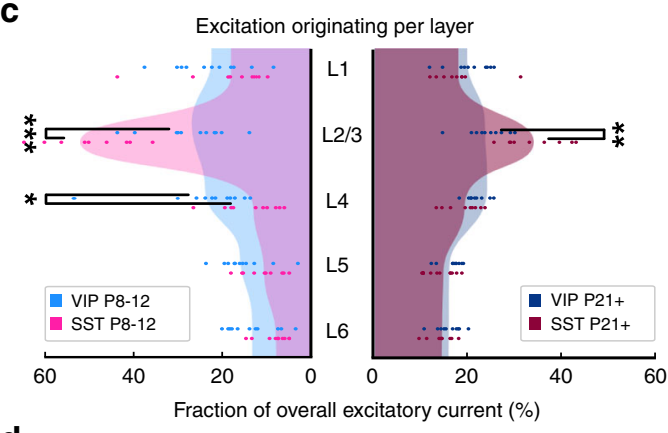

d
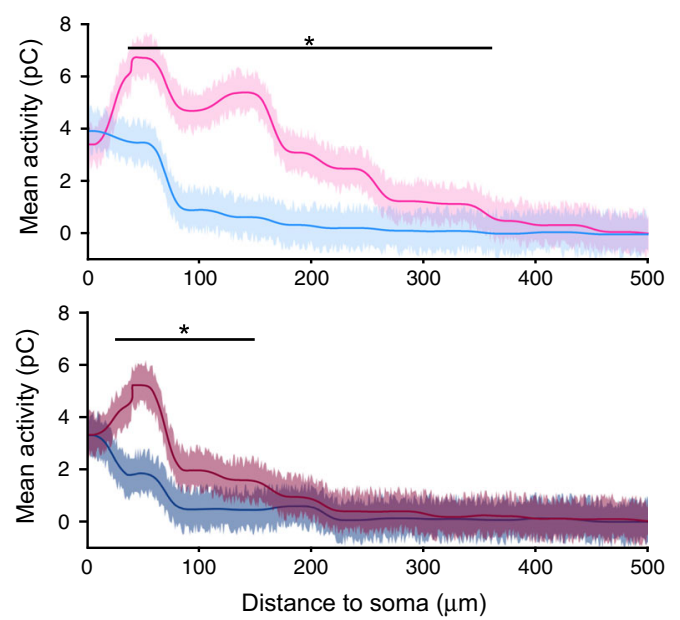

Fig. 3 Distinct functional connectivity motifs onto superficial VIP ${ }^{+}$and $\mathbf{S S T}{ }^{+}$cortical interneurons. a Left: a graphic representation of photostimulation celibration with a patched putative pre-synaptic neuron within a neocortical slice indicated with layer boundaries (L1-6). Illumination calibration were done so that the paradigm successfully evoked a short train of APs in the putative pre-synaptic partners. AP discharge is displayed to the right, with the peak of the first AP until after the last AP creating a time-window used for future analysis of evoked currents. Right: schematic representation of photostimulationbased mapping of incoming currents onto interneurons through whole-cell patch-clamp recordings. Overlaid is a schematic of the $20 \times 20$ grid, indicating the quadrants that can be photostimulated. Two of the AP discharges of the interneuron is shown to the right, with one not eliciting (1) and one eliciting (2) a post-synaptic response. The time-window acquired during calibration is used to register and analyse the currents. b Top: example traces of voltage responses to square hyperpolarizing and depolarizing currents pulses, $\Delta+20 \mathrm{pA}$. Bottom: Heatplot representations of normalized evoked excitatory current integral (in PC), recorded over development (P8-12 and P21+) from VIP+ (left) and SST+ (right) IN while perfoming glutamate uncaging in a grid pattern (VIP P8-12: 11 cells, VIP P21+ and SST P8-12: 10 cells, SST P21+: 9 cells). c plots of excitatory input onto VIP+ and SST+ cells (individual dots) averaged per lamina and normalized to average overall excitation within the field of view. The grand average of all cells per group is depicted as a continuous filled wave and compared within age groups. One data point has been excluded for display purposes only. Same number of cells as in b. Statistics: two-sided Mann-Whitney $U$ test, $p(L 2 / 3$ P8-12 $)=4.909 \times 10^{-4}, p(L 4$ P8-12) $=0.018, p(L 2 / 3$ P21+ $)=0.0015$. d Mean evoked excitation originating from within L1-3 and plotted as a fuction of lateral distance from either side of the recorded cells somata. Same number of cells as in b. Statistics: two tailed $t$-test $\left({ }^{\star} p<0.05,{ }^{\star \star} p<0.01,{ }^{\star \star \star} p<0.001\right)$. Source data are provided as a Source data files.

stimulation in a grid pattern in wS1-containing brain slices while measuring evoked postsynaptic excitatory and inhibitory currents

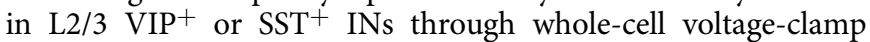
recordings at $-45 \mathrm{mV}$ (Fig. 3a). With this approach, we mapped out the origin as well as the strength of incoming cortical connections at the two developmental stages investigated (P8-12 and $\mathrm{P} 21+$ ). We found that ${\mathrm{L} 2 / 3 \mathrm{SST}^{+}}^{+}$INs receive the majority of their excitatory input from within their own layer and that this is the case both before and after P14. These results fit well with the laminar patterns of excitatory inputs described for the L2/3 SST/ $\mathrm{CR}^{+}$cells in adult mice ${ }^{30}$. The $\mathrm{VIP}^{+}$cells on the other hand receive almost equal amounts of excitatory inputs from all layers, with a decrease in L5/6 (Fig. 3c). Because the photostimulation was carried out over a large field of view we were able to assess lateral activity originating from up to $500 \mu \mathrm{m}$ away from either side of the IN cell body and therefore inputs coming from outside the resident column. To compare our glutamate uncaging results to the rabies virus mapping, inputs originating in the superficial layers (L1-3) were plotted in relation to their lateral distance from the recorded cell. In agreement with the rabies virus findings, the analysis showed that prior to $\mathrm{P}^{4} \mathrm{SST}^{+}$INs receive excitatory inputs from more lateral sources compared to $\mathrm{VIP}^{+}$cells (Fig. 3d). Even though distal inputs onto both cell types seem to have undergone pruning at $\mathrm{P} 21+$ (Fig. 3d compare upper and lower panel), $\mathrm{SST}^{+}$INs still receive a higher proportion of excitatory inputs from further away compared to $\mathrm{VIP}^{+}$INs (Fig. 3d). Interestingly, the $\mathrm{VIP}^{+}$INs receive more inhibition than the $\mathrm{SST}^{+}$INs from L2/3 both before and after P14, acting almost in an opposite fashion to the mapped incoming excitation (Fig. S5f, g). Because glutamate uncaging could activate the receptors on the patched cell directly, and holding the cell at -45 $\mathrm{mV}$ does not allow for a proper estimation of purely excitatory responses, a new set of photostimulation experiments was carried out in voltage-clamp mode at $-70 \mathrm{mV}$, first without and then in 
the presence of tetrodotoxin (TTX). By subtracting the direct responses (in the presence of TTX) from the evoked excitatory ones, recorded before the addition of the drug, currents evoked exclusively from pre-synaptic neurons could be calculated (Fig. S5h, i). The analysis of this new dataset showed that the $\mathrm{SST}^{+}$INs do indeed receive excitation from more distant sources compared to the $\mathrm{VIP}^{+}$cells within the superficial layers (Fig. $3 \mathrm{~d}$ ).

The anatomical tracing data together with the functional incoming excitation data suggest that $\mathrm{SST}^{+}$INs receive more lateral excitation compared to $\mathrm{VIP}^{+}$cells at both developmental time points. This provides a plausible mechanism for the stronger activation of SST ${ }^{+}$INs by multi-whisker stimulation both before and after P14. Although $\mathrm{VIP}^{+}$cells only respond stronger to multi- compared to single-whisker stimuli before P14, they do not show striking changes in cross-columnar inputs across the two ages. Nonetheless, small changes in connectivity can have a large functional effect given the slow membrane time constant (Fig. 3b) and high membrane resistance of immature VIP ${ }^{+}$cells (Fig. S5a). Therefore the developmental changes observed in $\mathrm{VIP}^{+}$cells for multi-whisker activation could be enabled by small changes in local cortical connectivity motifs. However, this does not preclude that $\mathrm{VIP}^{+}$INs might also undergo changes in long-range thalamic connectivity.

Rearrangement of thalamic inputs onto superficial $\mathrm{VIP}^{+}$and SST $^{+}$interneurons during development. Since the observed inter-barrel connectivity motifs are not able to fully explain the developmental changes in single- versus multi-whisker responses seen in the in vivo $\mathrm{Ca}^{2+}$ imaging data, we turned our attention to bottom-up inputs coming from the thalamus, labeled by our rabies tracings. The analysis was facilitated by the preservation of the $3 \mathrm{D}$ architecture of the thalamus in the cleared whole-brain dataset. Interestingly, we found that before $\mathrm{P} 14$, both $\mathrm{VIP}^{+}$and $\mathrm{SST}^{+}$INs had many presynaptic cells in the thalamus (Fig. $4 \mathrm{a}, \mathrm{c}$ and Movies S1 and S3), mainly located in the ventral posteromedial nucleus of the thalamus (VPM), but also in the posterior complex (PO), a higher-order nucleus primarily innervating wS1 L1-3 and L5a, as well as $\mathrm{wS}^{31}$ (Fig. 4a, c, e and Movies S5 and S7). Unexpectedly, we found a clear shift in the thalamic nuclei providing input to the $\mathrm{SST}^{+}$INs after P14. In contrast to prior P14, very few or no pre-synaptic partners were found in the VPM or PO, while many cells were labeled in the Ventro-Medial nucleus (VM) and the Ventro-Anterior nucleus (VAL) of the thalamus (Figs. 4d, e and S7, Movies S4 and S8). Interestingly, after P14 the $\mathrm{VIP}^{+}$INs did not seem to have any pre-synaptic partners in the thalamus (Fig. 4b and Movies S2 and S6). This is in contrast to studies that have shown functional input from the thalamus onto $\mathrm{VIP}^{+}$cells in the adult cortex ${ }^{18,19,32}$. Since it is widely known that the rabies virus does not spread to all the presynaptic partners of a cell ${ }^{33-35}$, and in our case this could also be augmented by reduced expression of the glycoprotein from the HTB mouse line ${ }^{36}$, we believe that this result reflects a reduction in thalamic inputs rather than a complete loss.

To examine this, we used two alternative approaches. First, an AAV HTB helper virus was co-injected with the rabies virus at P15 in VIPCre mice. Using this approach, we indeed detected a low number of pre-synaptic cells in the VPM and the PO (Fig. S7). This would suggest that there is indeed pruning of thalamo-cortical inputs onto VIP cells, which we further tested using staining for the vesicular glutamate transporter 2 (VGlut2), a marker for thalamo-cortical terminals in the two age groups.

The analysis showed that prior to $\mathrm{P} 14, \mathrm{VIP}^{+}$INs had significantly more VGlut2 puncta onto their dendrites compared to $\mathrm{SST}^{+}$INs, whereas after P14 the number of appositions onto $\mathrm{VIP}^{+}$INs dropped significantly (Fig. 4f), evening out the difference between the two cell types. These histological results support the data obtained with the rabies-based mapping, and suggest that upon the onset of active whisking thalamic input onto $\mathrm{VIP}^{+}$INs is strongly reduced.

Overall, our data suggest a model by which the $\mathrm{VIP}^{+}$IN preference for multi-whisker stimulation before P14 is mainly supported by strong thalamo-cortical inputs that come from both VPM and PO. These inputs are significantly reduced after the onset of active whisking, together with the $\mathrm{VIP}^{+}$INs response to multi- versus single-whisker stimulation (Fig. 5). For the $\mathrm{SST}^{+}$ INs on the other hand, the preference for multi-whisker stimulation, both before and after P14 would be supported by the strong lateral inter-barrel connectivity, with a contribution of direct VPM and PO-derived excitation prior to P14 (Fig. 5).

\section{Discussion}

This study investigates the engagement of $\mathrm{VIP}^{+}$and $\mathrm{SST}^{+}$INs by sensory stimuli, and the underlying circuits they are embedded in before and after the onset of active whisking (P14).

We initially hypothesized that in the juvenile mouse wS1, $\mathrm{VIP}^{+}$ and $\mathrm{SST}^{+}$cells would be differentially activated by a singleversus a multi-whisker deflection based also on published reports ${ }^{11,20}$. By testing the activation pattern of the same population of INs for the two types of stimuli, we found support for our hypothesis. $\mathrm{VIP}^{+} \mathrm{INs}$ at $\mathrm{P} 21+$ responded in the same manner whether single or multiple whiskers were deflected, even though prior to P14 they responded much stronger to the multiwhisker stimulus. $\mathrm{SST}^{+}$cells at $\mathrm{P} 21+$ on the other hand responded stronger to the multi-whisker stimulus, which was also the case before P14. Even though in this study we have not directly examined the connectivity between these two interneuron types, recently published work in the adult mice showed strong layer-dependent effects of channelrhodopsin-based activation of $\mathrm{VIP}^{+}$neurons ${ }^{20}$. Specifically, the authors reported that L2-4 excitatory neurons did not increase their firing rate upon $\mathrm{VIP}^{+}$ neuron activation, suggesting that the recruitment of the VIP-SST inhibitory loop is layer- and most likely also stimulus-dependent. On the other hand, the same study suggests that upon active whisking and stimulation of multiple whiskers, $\mathrm{SST}^{+}$cells are strongly activated, whereas $\mathrm{VIP}^{+}$INs are inhibited with a delay that matches the activation of $\mathrm{SST}^{+}$neurons. These results are in line with the reciprocity of the connections between the two IN types $^{12}$, and together with our data suggest that the di-synaptic disinhibitory VIP-SST connection is engaged in a dynamic manner, with one cell inhibiting the other, the directionality of which is context-dependent.

Developmentally, the robust activation of both $\mathrm{VIP}^{+}$and $\mathrm{SST}^{+}$ INs prior to P14 by the multi- compared to the single-whisker stimulus, suggests that even if the connectivity between $\mathrm{VIP}^{+}$and $\mathrm{SST}^{+}$cells is present at this time point, it seems to be overridden by either local or long-range thalamic inputs onto both IN types.

Interestingly, we found striking developmental changes in the $\mathrm{Ca}^{2+}$ dynamics of the two INs, suggesting that $\mathrm{VIP}^{+}$and $\mathrm{SST}^{+}$ cells undergo significant maturation around the time the mouse starts to actively whisk. First, we found that before P14 the amplitude of whisker-stimulation-evoked $\mathrm{Ca}^{2+}$ activity is much lower than that of spontaneous events, suggesting that evoked responses have a different origin to spontaneous ones or that the former are being actively decreased upon sensory stimulation during this developmental period. Both $\mathrm{VIP}^{+}$and $\mathrm{SST}^{+}$cells receive stronger and more widespread inhibitory inputs at P8-12 which could potentially decrease the amplitude of somatic $\mathrm{Ca}^{2+}$ transients through GABAA and/or GABAB receptor-mediated mechanisms. Furthermore, evoked $\mathrm{Ca}^{2+}$ transients recorded before $\mathrm{P} 14$ are slower and more prolonged compared to both spontaneous activity 
a

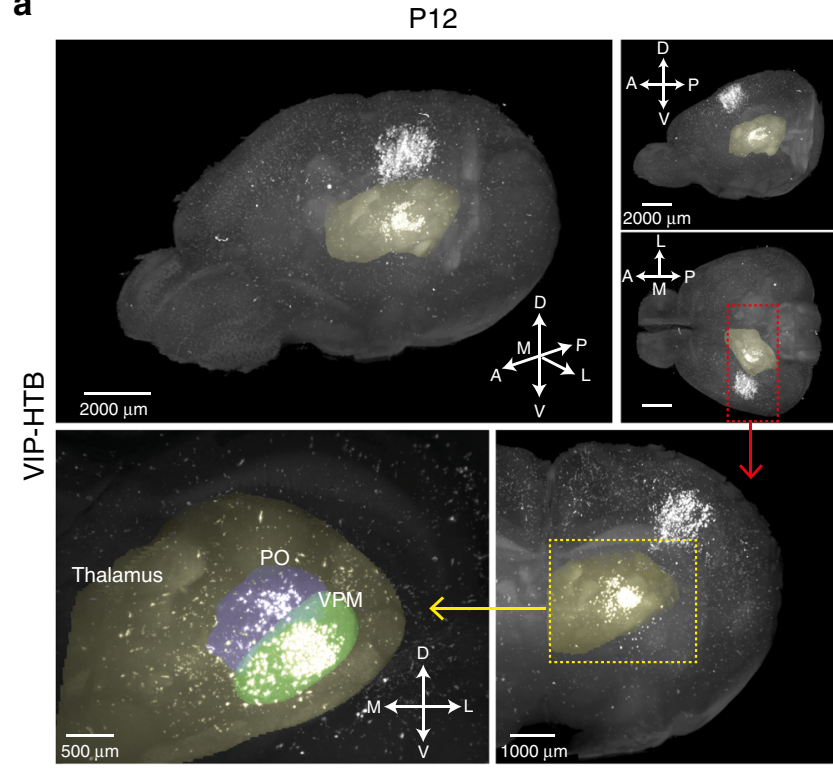

C
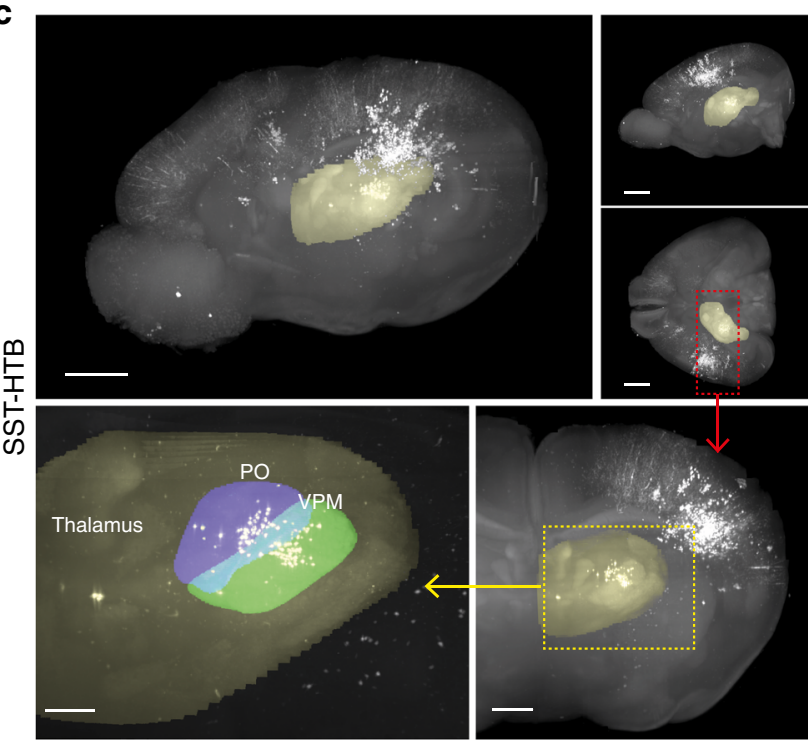

b
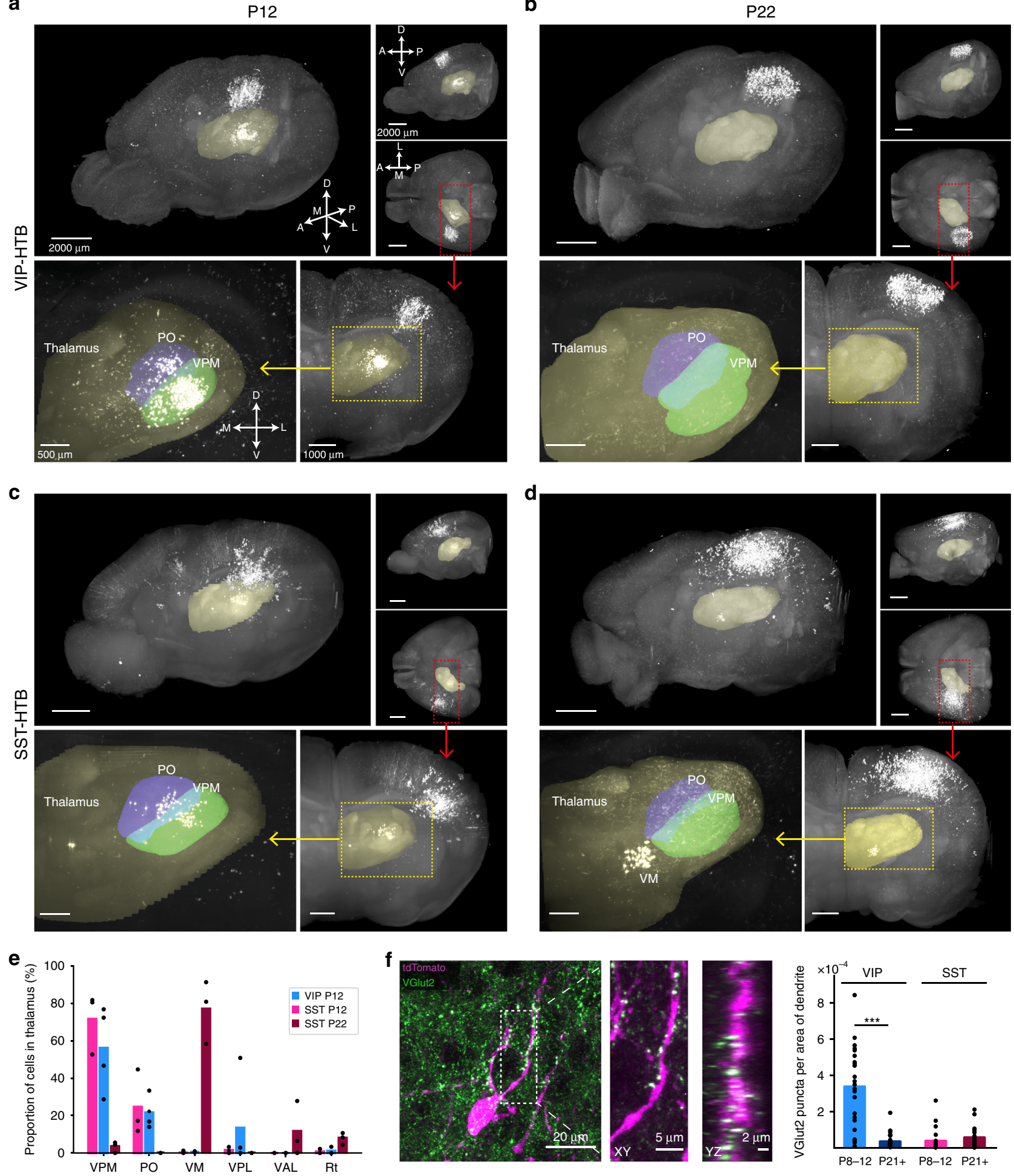
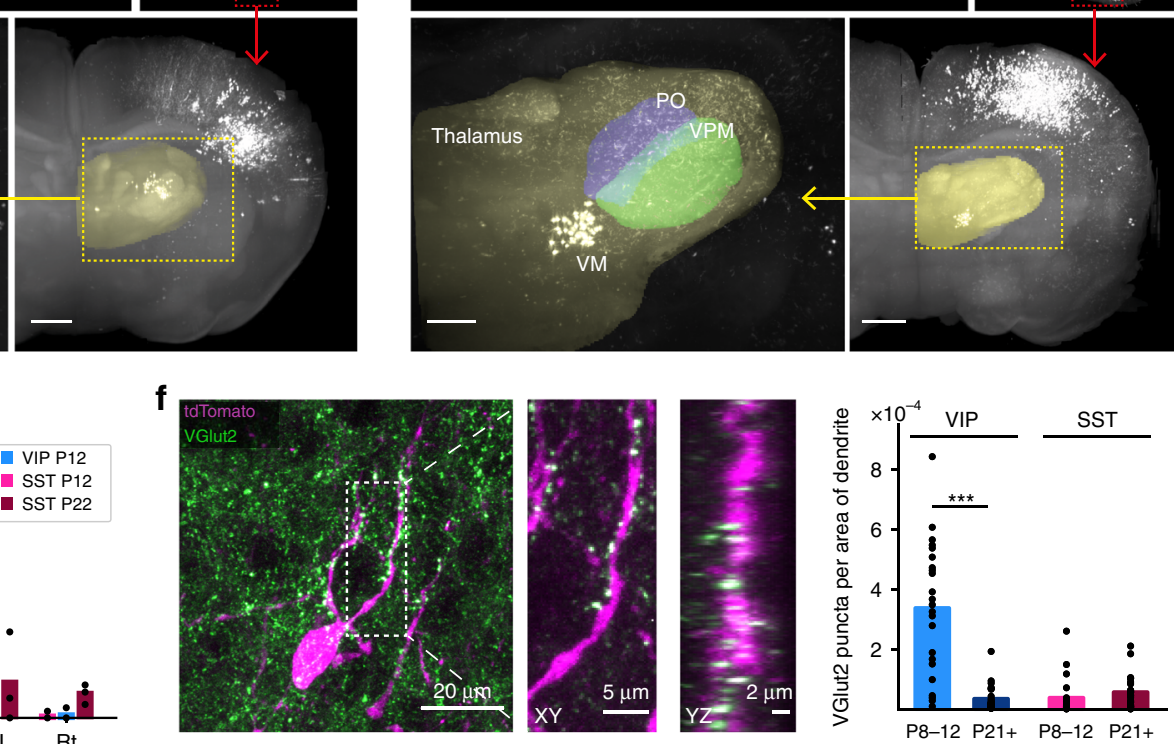

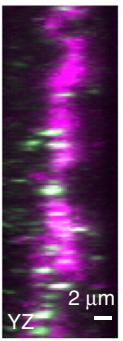

Fig. 4 Rearrangement of thalamic inputs onto superficial VIP ${ }^{+}$and $\mathbf{S S T}{ }^{+}$interneurons during development. a-d Top panel: representative examples of cleared rabies injected brains seen from an oblique, top-down and side angle. The thalamus is highlighted in yellow. The red square indicates the area projected in bottom panel. Bottom panel: Coronal view of maximum intensity projection of the thalamus. Left: overview. Right: zoom-in with VPM (green) and PO (blue) highlighted. $N=3$ brains for SST; $N=4$ brains for VIP P12). e Quantification of pre-synaptic cells in different thalamic nuclei normalized to the total number of cells in the thalamus. VIP P22 is not included because no cells were found in the thalamus. Same number of brains as in a-e. $\mathbf{f}$ Example of VGlut2 staining (green) in L2/3 of a wS1 section from a VIPCre-tdTomato animal at P9. The dotted square indicates the zoomed-in part on the right, which shows a close-up of puncta appositions in $x y$ and $y z$ direction. The graph shows quantification of VGlut2 puncta on tdTomato-positive dendrites. The number of appositions in every picture is normalized to the area of tdTomato positive dendrites in the same picture. ( $N=3$ brains per group; VIP P8-12: 23 images, VIP P21+: 27 images, SST P8-12: 21 images SST P21+: 28 images. Statistics: two-sided Mann-Whitney U test, $p($ VIP $)=2.492 \times 10^{-7}$. Source data are provided as a Source data file. 
P8-12
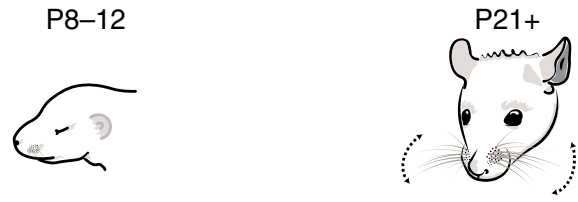

L1

$\mathrm{L} 2 / 3$

L4

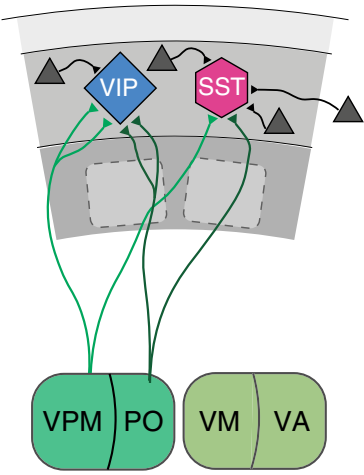

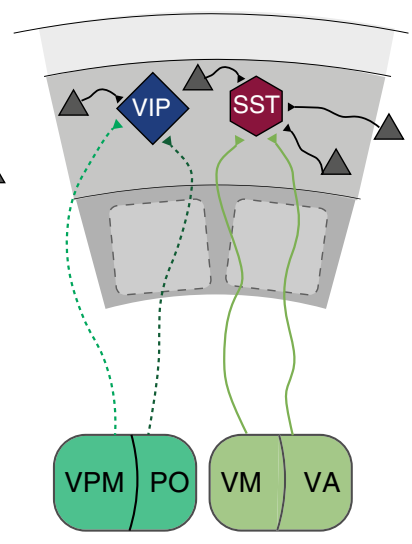

Fig. 5 Schematic of developmental input rearrangement onto superficial VIP $^{+}$and SST ${ }^{+}$interneurons. Local connectivity: $\mathrm{SST}^{+}$INs receive excitatory inputs (gray triangles) from more distal source within the barrel cortex than $\mathrm{VIP}^{+}$cells both before and after P14. Bottom-up inputs: Before $\mathrm{P} 14$ both $\mathrm{VIP}^{+}$and $\mathrm{SST}^{+}$cells receive the majority of their thalamic inputs from the VPM and PO nuclei of the thalamus, with the VIP+ cells showing more thalamic synapses on their dendrites. After P14 thalamic input onto VIP+ cells is strongly reduced while the input onto SST+ INs is shifted to the VM and VA thalamic nuclei.

at the same age and evoked activity after P14. There are a number of potential mechanisms that could explain changes in evoked transients over time such as developmental changes in action potential speed leading to a slower intracellular $\mathrm{Ca}^{2+}$ rise in the cytoplasm or altered buffering and/or extrusion capacity of the cells. Nevertheless, the fact that spontaneous $\mathrm{Ca}^{2+}$ signals before P14 do not show the same dynamics as the evoked suggests that the prolongation of the signal is not due to a cell-intrinsic mechanism, but rather to the network activity engaged upon sensory stimulation. For example, reverberating networks could underlie the generation of multiple action potentials spread across time and lead to slower $\mathrm{Ca}^{2+}$ transients. This may be especially the case when multiple whiskers are deflected. In this case, the signal would activate the VPM, which through direct connections activate the VIP ${ }^{+}$and SST + INs. In addition to this early activation, there would be a later wave of activity onto INs via the engagement of a parallel pathway, consisting of a loop between these INs in $\mathrm{wS} 1$ and $\mathrm{PO}^{37,38}$, leading to the prolonged $\mathrm{Ca}^{2+}$ transient component. Furthermore, our wS1 distance-dependent anatomical and functional connectivity analysis show that in neonatal mice there is strong widespread cortical input in both cell types which could also contribute to the prolonged $\mathrm{Ca}^{2}$ + transients before P14. Nevertheless, excitation coming from lateral cross-barrel domains is stronger onto $\mathrm{SST}^{+}$compared to $\mathrm{VIP}^{+}$ cells both before and after P14, whereas inhibition from within layers $2 / 3$ is stronger onto $\mathrm{VIP}^{+}$cells. This higher inhibition onto the latter cells, could be originating from multiple types of presynaptic GABAergic cells, including other $\mathrm{VIP}^{+}$cells, reelinexpressing $\mathrm{INs}^{39}$ or $\mathrm{SST}^{+}$cells, as suggested by $\mathrm{Yu}$ et $\mathrm{al}^{20}$.

At the same time, we find a striking developmental change in bottom-up thalamic inputs onto $\mathrm{SST}^{+}$INs. Whereas prior to active whisking they receive the majority of inputs from the sensory-related VPM and PO nuclei, after the onset of active whisking their thalamic inputs shift to motor-related nuclei, such as the VM and the VAL. A study by Wall et al., that combined AAV helper virus and monosynaptic rabies virus to trace the inputs onto inhibitory INs in the adult S1, showed that the VPM and $\mathrm{PO}$ strongly project to $\mathrm{SST}^{+}$INs, while the motor-related nuclei only showed few presynaptic cells ${ }^{40}$. However, it is important to note that in our experiments we get very few starter cells in the main thalamo-recipient layer (L4) compared to the abovementioned study. In addition, due to the usage of wholebrain clearing and the autofluorescence of the thalamic barreloids (VPM), we have been able to very precisely assign cells to different thalamic nuclei, which could have been mis-assigned to more rostral VPM using histological approaches.

Along the same lines, another study that reported the in vivo activity of $\mathrm{SST}^{+}$neurons in adult animals showed that they do not receive strong inputs from the $\mathrm{VPM}^{20,32}$. The authors suggested that $\mathrm{SST}^{+}$INs may rather be recruited by local excitatory inputs, which fits well with our cortical connectivity results onto these interneurons. However, we can certainly not exclude that some of the $\mathrm{SST}^{+}$activation comes from different thalamic nuclei such as VM and VAL. These nuclei are in fact known to project to the striatum, L1 of motor-related cortical areas, the cingulate as well as the prefrontal cortex ${ }^{41-45}$. A proportion of VM cells also have axonal collaterals to S1, the target specificity of which was until this study unknown ${ }^{46,47}$. AAV tracings from the Allen Mouse Brain Connectivity Atlas (http://connectivity.brain-map. org/projection) show that axonal projections from the VM exclusively target L1 of the somatosensory cortex. This strongly suggests that the connection we uncover with the rabies tracing is formed between $\mathrm{VM}$ and $\mathrm{L} 2 / 3 \mathrm{SST}^{+}$cells as they are known to extend their dendrites into $\mathrm{L}^{48}$. Furthermore, the VM is activated during whisking events, as assessed by in vivo photometry with GCaMP6 ${ }^{49}$, suggesting that it is involved in reporting whisker-related information, which it could potentially pass on to the superficial $\mathrm{SST}^{+}$INs.

Notwithstanding the underlying mechanisms, both $\mathrm{VIP}^{+}$and $\mathrm{SST}^{+}$INs show greater responses upon multi- compared to single-whisker stimulation before P14. Together with our anatomical data, this indicates that the functional impact of bottom-up input onto $\mathrm{VIP}^{+}$cells decreases and becomes more specific after P14. We would speculate that this decrease coincides with the emergence of top-down modulation from wM1 to allow these cells to act as coincident detectors between active whisking and a touch event of an individual whisker. The $\mathrm{SST}^{+}$INs on the other hand would be preferentially activated if multiple whiskers are deflected simultaneously. We would argue that the ability of $\mathrm{SST}^{+}$ INs to differentiate between single- and multi-whisker stimuli in the juvenile $\mathrm{wS} 1$, suggests that these cells act antagonistically to the activation of $\mathrm{VIP}^{+}$cells, depending on how each whisker is deflected in relation to their neighboring ones. We would speculate that upon complex navigation or object exploration, in some parts of the $\mathrm{wS}_{\mathrm{SST}} \mathrm{SS}^{+}$activation would take over, while in others $\mathrm{VIP}^{+}$cells would engage more. Therefore, our results support a model that upon the onset of active whisking, $\mathrm{VIP}^{+}$and $\mathrm{SST}^{+}$ INs diversify their functions to be able to register multiple aspects of the environment and hence help facilitate appropriate tactile perception.

\section{Methods}

Animals. All animal experiments were approved by the Cantonal Veterinary Office Zurich and the University of Zurich. Animals were housed in a 12-h reverse darklight cycle (7 a.m. to 7 p.m. dark) at $24^{\circ} \mathrm{C}$ and variable humidity. Animal lines used

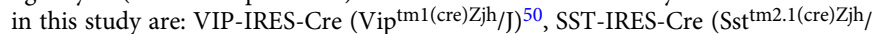

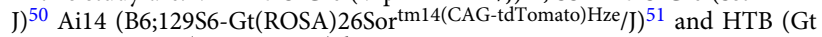

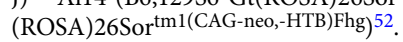

Animal surgery and preparation for in vivo imaging experiments. We used 6 VIPCre-tdTomato and 6 SSTCre-tdTomato mice ( 7 males, 5 females) at ages 
ranging from $\mathrm{P} 8$ to $\mathrm{P} 41$ for two-photon $\mathrm{Ca}^{2+}$ imaging. As described previously ${ }^{21}$, mice were sedated with chlorprothixene $(0.1 \mathrm{~g} / \mathrm{kg}$, intraperitoneal (i.p.); SigmaAldrich Chemie GmbH, Buchs, Switzerland) and lightly anesthetized with urethane throughout the experiment $(0.25-0.5 \mathrm{~g} / \mathrm{kg}$, i.p.). Atropine $(0.3 \mathrm{mg} / \mathrm{kg}$; SigmaAldrich Chemie GmbH, Buchs, Switzerland) and dexamethasone $(2 \mathrm{mg} / \mathrm{kg}$; aniMedica GmbH, Senden-Bösensell, Germany) were injected subcutaneously (s.c.) 30 mins after induction of anesthesia to reduce saliva secretion and prevent edema. Body temperature was kept at $37^{\circ} \mathrm{C}$ using a heating pad. Hydration levels were maintained by s.c. injections of Ringer-lactate (Fresenius Freeflex; Fresenius Kabi AG, Oberdorf, Switzerland) and depth of anesthesia was checked throughout the experiment by pinching the forepaw and checking for reflex. To stabilize the animal, a custom-built head plate was glued to the skull over the left hemisphere using dental cement (Paladur, Heraeus Kulzer GmbH Hanau, Germany; Caulk Grip Cement for electrophysiology).

Using a sharp razor blade a small cranial window of $\sim 1.5 \times 1.5 \mathrm{~mm} 2$ was opened above the center of the mapped barrel columns and superfused with Ringer's solution (in mM: $145 \mathrm{NaCl}, 5.4 \mathrm{KCl}, 10 \mathrm{HEPES}, 1 \mathrm{MgCl}_{2}, 1.8 \mathrm{CaCl}_{2} ; \mathrm{pH}$ 7.2 adjusted with $\mathrm{NaOH}$ ). Care was taken not to damage the dura or surface blood vessels in young animals. In animals older than P20, the dura was removed to prevent blockage of the glass pipette tip during $\mathrm{Ca}^{2+}$ indicator loading.

Intrinsic optical imaging. The principal whisker-related barrel column was identified using optical imaging of intrinsic signals. The cortical surface was visualized through the intact bone by surface application of normal Ringer's solution and a glass coverslip placed on top. The skull surface above the barrel cortex was left intact for animals younger than P12, but thinned in older animals. Reference images of the cortical blood vessel pattern were visualized by a $546-\mathrm{nm}$ LED to enhance contrast. Functional maps of the target barrel column C2 were obtained by shining red light ( $630 \mathrm{~nm}$ LED) on the cortical surface while stimulating the $\mathrm{C} 2$ whisker with a galvanometer $\left(10 \mathrm{~Hz}\right.$ for $2 \mathrm{~s}$ at $1140^{\circ} / \mathrm{s}$ amplitude in rostro-caudal direction ${ }^{21}$ ). Reflectance images were collected through a $4 \times$ objective with a CCD camera (Scientifica SciCam Pro; 14-bit; 2-by-2-pixel binning, $680 \times 512$ binned pixels at $31 \mathrm{fps}$ ). Functional intrinsic signal images were computed as fractional reflectance changes relative to the pre-stimulus average (average of 10 trials). The intrinsic signal image obtained for the $\mathrm{C} 2$ barrel column was then mapped to the blood vessel reference image and used to guide the location of the craniotomy and $\mathrm{Ca}^{2+}$ imaging.

Galvanometer-driven whisker stimulation. Whisker stimulation was performed with a two galvanometer-driven stimulation ${ }^{21}$. One stimulation fiber was attached to the $\mathrm{C} 2$ whisker considering variations in resting position angles and relative anterior-posterior shifts. The second stimulator was positioned closer to the whisker pad and a small holder perpendicular to the fiber arm was added for multiwhisker stimulation. For single principal whisker stimulation only the first fiber was moved, whereas for multi-whisker deflection, both the fibers were moved at the same time. The stimulation fibers were fixed and secured with Plasticine on top of a custom-built holder plate and secured and translated with a micro-manipulator. Deflections were applied in the rostro-caudal direction and one single pulse consisting of a phase-shifted $100 \mathrm{~Hz}$ cosine with $1140 \%$ peak velocity was applied through either one or both galvanometer-driven stimulators.

In vivo two-photon calcium imaging. Neuronal ensembles in superficial layers of the principal whisker barrel field mapped by intrinsic signal imaging were bolusloaded with the AM ester form of Oregon Green BAPTA-1 by pressure injection (OGB-1; 1 mM solution in $\mathrm{Ca}^{2+}$-free Ringer's solution; 2-min injection at 150-200 $\mu \mathrm{m}$ depth) as described previously ${ }^{53}$. The craniotomy was then filled with agarose (type III-A, 1\% in Ringer's solution; Sigma) and covered with an immobilized glass plate. Two-photon $\mathrm{Ca}^{2+}$ imaging was performed with a Scientifica HyperScope two-photon laser scanning microscope one hour after bolus loading using a Ti: sapphire laser system at $900 \mathrm{~nm}$ excitation (Coherent Chameleon; 120 femtosecond laser pulses). Two-channel fluorescence images of $256 \times 128$ pixels at 11.25 $\mathrm{Hz}$ (HyperScope galvo-mode) were collected with a 16x water-immersion objective lens (Nikon, NA 0.8). In all, 3-5 separate spots (i.e. Fig. 1c top row) have been imaged per animal. Per imaging spot, both a 300-s long continuous recording of spontaneous activity as well as 10 trials of 20s-long evoked activity recorded for each of single- and multi-whisker stimulation paradigms. Data acquisition was controlled by ScanImage ${ }^{54}$. Duration of $\mathrm{Ca}^{2+}$ imaging recordings varied between 3 and $4 \mathrm{~h}$.

Analysis of calcium imaging data. $\mathrm{Ca}^{2+}$ imaging data were imported and analysed using routines custom-written in MATLAB. First, fluorescence image timeseries for a given region were concatenated. The concatenated imaging data was then aligned using a cross-correlation based subpixel registration algorithm ${ }^{55}$ to correct for translational drift (registered on red tdTomato channel and transferred to OGB-1 channel). Average intensity projections of the imaging data were used as reference images to manually annotate regions of interest (ROIs) corresponding to individual neurons. Neurons with somata partly out-of-focus were not included. $\mathrm{Ca}^{2+}$ signals were expressed as the mean pixel value of the relative fluorescence change $\Delta F / F=\left(F-F_{0}\right) / F_{0}$ in each given ROI. $F_{0}$ was calculated as the bottom $5 \%$ of the fluorescence trace. Neuropil patches surrounding each neuron is defined by all pixels not assigned to a neuronal soma or astrocyte of the corresponding neuron ROI annotation ${ }^{56}$ (A disk shaped region around the neuron of interest with excluding any intersecting neighboring neuronal ROI). Neuropil correction is performed as $F_{\text {corrected }}=F_{\text {neuron }}-\alpha^{*} F_{\text {neuropil. }} \alpha$ is estimated for each imaging spot separately using the formula $F_{\text {blood_vessel }} / F_{\text {surrounding_neuropil }}{ }^{57}$. For each stimulus, the evoked responses of 10 trials were analyzed and the response magnitude expressed as the mean of the evoked $\Delta F / F$ integral (\%·s; integral of the first $8 \mathrm{~s}$ response starting at stimulus onset). Pearson's correlation coefficients of sensory-evoked responses for any two neurons at zero lag were calculated for each single trial evoked calcium traces in a $17.4 \mathrm{~s}$ window starting from stimulus onset. Spontaneous correlations were calculated by averaging correlations of 1000 randomly segmented 17.4-s long pieces from a $300 \mathrm{~s}$ long spontaneous recording, to get rid of the trace length-dependent correlation fluctuations for the comparison of spontaneous and evoked correlations. To detect spontaneous events, we first ran a peak finding algorithm (in MATLAB) and afterwards manually checked each peak to verify it is an actual event. Our definition of an event was a peak followed by an (bi)exponential decay with a 1-2 s flat baseline. When the signal-to-noise (SNR) ratio in the data is relatively high, it is easier to detect such events, however, it gets harder as the SNR gets lower. In the juvenile dataset, SNR was significantly higher than the neonatal dataset, making the detection of the events in the latter more difficult. In order to fit the calcium responses properly in all groups we had to use a double exponential fit, after which we calculated the weighted decay tau:

$\tau=\left(f \cdot a^{*}(-1 / f \cdot b)+f . c^{*}(-1 / f \cdot d)\right) /(f \cdot a+f . c)$ where $f=a^{*} \exp \left(b^{*} t\right)+c^{*} \exp \left(d^{*} t\right)$ using Matlab curve fitting toolbox functions.

Decoders. Three different decoders were trained either for the multi- or singlewhisker stimulation paradigm, to quantify the decoding capacities of the SSTs and the VIPs at P8-P12 and P21+. The decoders used were random forest, naïve Bayes and Error-correcting output codes (ECOC) that classified a cell type into one out of two groups based on baseline-subtracted average stimulus response trials ${ }^{58}$. Each classifier was trained 1000 times by randomly setting $70 \%$ of the data for training (Matlab function TreeBagger, with parameters $N_{\text {trees }}=50$, minleaf $=5$; fitcnb and fitcecoc, respectively). For each trained set, $30 \%$ of the remaining data was used to calculate classification accuracy.

Acute slice electrophysiology. Whole-cell patch-clamp electrophysiological recordings were performed on either SSTCre-tdTomato or VIPCre-tdTomato cells in L2/3 of the wS1, in acute slices prepared from P8-12 or P21+ animals.

Animals were anesthetized, decapitated, the brain extracted and transferred to $4{ }^{\circ} \mathrm{C}$ physiological Ringer's solution (aCSF), of the following composition (mM): $125 \mathrm{NaCl}, 2.5 \mathrm{KCl}, 25 \mathrm{NaHCO}_{3}, 1.25 \mathrm{NaH}_{2} \mathrm{PO}_{4}, 1 \mathrm{MgCl}_{2}, 2 \mathrm{CaCl}_{2}$ and 20 glucose. The brain was then glued to a stage and cut into $300 \mu \mathrm{m}$-thick coronal slices using a vibratome (VT 1200S, Leica). The slices recovered in room temperature aCSF for $30 \mathrm{~min}$ before recording. The slices were then placed in the recording chamber of an upright microscope (Axioscope $2 \mathrm{FS}$, Zeiss) and superfused with $32{ }^{\circ} \mathrm{C}$ oxygenated $\left(95 \% \mathrm{O}_{2}\right.$ and $\left.5 \% \mathrm{CO}_{2}\right)$ aCSF at a rate of $2-3 \mathrm{ml} / \mathrm{min}$. The microscope was equipped with immersion differential interference contrast (DIA) and the following objectives were used to visualize the cells $(10 \times / 0.3$, Olympus and $40 \times / 0.8$, Zeiss). A CMOS camera (optiMOS, QImaging) was attached to the scope to visualize the slice and cells through a computer screen. A white-light source (HAL 100, Zeiss) and a LED based excitation source (Polygon400, Mightex Systems) in combination with a tdTomato filter set (set $43 \mathrm{HE}$, Zeiss, Excitation 550/25, Emission 605/70) were used to locate the fluorescent INs. Patch pipettes were pulled from borosilicate glass capillaries $(1.5 \mathrm{OD} \times 0.86 \mathrm{ID} \times 75 \mathrm{~L} \mathrm{~mm}$, Harvard Apparatus) at a resistance of 4-6 $\mathrm{M} \Omega$.

For recordings of intrinsic electrophysiological properties and photostimulation-evoked currents, Clampex was used (v10.7.0.3, Molecular Devices 2016). The recording pipettes were filled with a solution containing the following (mM): 135 potassium D-gluconate, $4 \mathrm{NaCl}, 0.3 \mathrm{Na}$-GTP, $5 \mathrm{Mg}$-ATP, 12 phosphocreatine-di-tris, $10 \mathrm{HEPES}, 0.0001 \mathrm{CaCl}_{2}$ (pH 7.25, mOsm 290). In all cases, $3 \mathrm{mg} / \mathrm{ml}$ biocytin (Tocris) was added in the recording solution and no extra pharmacology was added. Access resistance was constantly monitored to ensure the quality and stability of the recording. The recorded data were accepted only if the initial series resistance was less than or equal to $25 \mathrm{M} \Omega$ and did not change by more than $20 \%$ throughout the recording period. Compensation was made for the pipette and cell capacitance.

We used a similar stimulation protocol as previously reported ${ }^{59,60}$. For each analyzed cell, passive and active membrane properties were recorded in currentclamp mode at $-65 \mathrm{mV}$ by applying a series of linearly increasing hyperpolarizing and depolarizing sub- and supra-threshold current steps $(500 \mathrm{~ms}, \Delta+20 \mathrm{pA})$. The analysis of intrinsic properties was done in Clampfit (v10.7.0.3, Molecular Devices 2016). Using this protocol, we analyzed the cells for five different parameters: For calculating the membrane resistance, we used the $R=\mathrm{V} / \mathrm{I}$ on hyperpolarizing pulses that did not lead to an activation of voltage-dependent conductances; Spike threshold was obtained as the inflection point of a rapid change in $\mathrm{dV} / \mathrm{dt}$ of the first spike evoked at the step protocol. The spike height was from the threshold voltage point to the maximum value of the peak. Spike width was calculated as the width at half of the maximum amplitude; After hyperpolarization amplitude was measured 
from spike threshold to the lowest occurrence point after the repolarization phase of the spike.

All intrinsic electrophysiology data presented in the paper are average.

In vitro calcium transient and AP correlation. Whole-cell patch-clamping of SSTCre-tdTomato $^{+}$neurons of L2/3 in wS1 was performed at P8-12 and P21+. Patch pipette was filled with $100 \mu \mathrm{M}$ OGB-1 and right after breaking into patched cell, an incubation period of $25 \mathrm{~min}$ took place to let the OGB-1 equilibrate within the cell body. A square pulse current was injected giving rise to a set number of APs $\left(\# 1,3,5,7\right.$, and 10), while simultaneously recording the evoked $\mathrm{Ca}^{2+}$ transient from the somata, using a Scientifica SliceScope two-photon laser microscope and a laser system at $900 \mathrm{~nm}$ excitation. Two-channel fluorescent images of $256 \times 128$ pixels at $11.25 \mathrm{~Hz}$ (HyperScope galvo-mode) were collected with a $40 \times$ waterimmersion objective lens. The $\Delta F / F$ of the gathered $\mathrm{Ca}^{2+}$ responses per a given number of APs were analyzed in terms of the $\mathrm{Ca}^{2+}$ transient max amplitude, decay tau, and integral. The Averages of these parameters were taken and compared between the two age groups, P8-12 and P21+.

Glutamate uncaging and data analysis. For photostimulation experiments, whole-cell patch-clamp recordings were performed on randomly selected INs in L2/3 and using a 10× objective lens (Olympus, NA 0.3, $1048 \times 1960 \mu \mathrm{m}$ field of view). For light stimulation, the tissue was digitally divided into 400 subregions making up a grid pattern, spanning all cortical layers. The tissue was submerged in oxygenated aCSF containing caged glutamate ( $295 \mu \mathrm{M}$ RuBi-Glutamate, Tocris) and photostimulation was performed at least three times at each spot, in a pseudorandom manner, using a high-power LED light source $(470 \mathrm{~nm}, 3300 \mathrm{~mW}$, Mightex Systems). Photostimulation parameters were calibrated beforehand so that the experimental paradigm successfully evoked a short train of $\sim 4$ APs in randomly selected putative pre-synaptic partners of the recorded cells. The average time of the evoked APs in putative pre-synaptic neurons were used as a window of analysis for the responses evoked within the patched INs (Fig. 3a). This time-window was used to capture majority of mono-synaptic activity and to minimize the presence of poly-synaptic. Although, when performing the glutamate uncaging, the activation of presynaptic partners through photostimulation never resulted in AP elicited currents within the postsynaptic patched cell, suggesting that the contribution of polysynaptic activity would be small. Recordings of synaptic activity were performed in voltage-clamp mode at $-45 \mathrm{mV}$ (to register both excitatory and inhibitory currents), with a sampling rate of $10 \mathrm{kHz}^{61-63}$.

Custom-written Matlab script was used to analyze the photostimulation evoked current data. Within the data gathered at $-45 \mathrm{mV}$, the excitatory currents were extracted by taking all activity registered below baseline (average of the signal within $500 \mathrm{~ms}$ before illumination) within the analysis window, while inhibitory currents were all activity above baseline.

For each recording, the field of view was segmented per layer and the average activity within each layer was normalized to the overall average activity within the whole field of view. Mann-Whitney $U$ test was carried out for all data points within each layer and comparison was made between lamina.

For the lateral activity analysis, the average activity within L2/3 was calculated per given distance from each recorded cells soma. The average was then calculated for all recordings within a given group. Two-tailed $t$-test was used to look for significance between groups and points with same given distance from soma.

The second set of photostimulation experiments were performed on SSTCretdTomato or VIPCre-tdTomato cells in L2/3 of the wS1, in acute slices prepared from P8-12 animals. This set of glutamate uncaging experiments was performed in same manner as described above but at $-70 \mathrm{mV}$, to allow to selectively detect only incoming excitatory currents to our recorded cells. When a first round of photostimulation had been carried out, $1 \mu \mathrm{M}$ TTX (Tetrodotoxin citrate, Tocris) was added to the circulating aCSF and $12 \mathrm{~min}$ after, the same glutamate uncaging experiment was performed. Through custom-written Matlab script we then subtracted the values obtained before from after TTX to calculate the extent of the synaptically evoked responses originating from pre-synaptic partners alone, rather than direct stimulation of the recorded cell. When performing this analysis only evoked activity originating from the superficial layers were considered.

Immunohistochemistry. For the immunohistochemistry experiments, the Ai14 reporter mouse line was combined with either VIPCre or SSTCre. The offspring were sacrificed at either P9-12 (before onset of active whisking) or at P21+ (after the onset of active whisking). In short, the animals were anesthetized before being transcardially perfused with 1x PBS followed by ice-cold 4\% PFA. The brains were then dissected and post-fixed in ice-cold $4 \%$ PFA for $1 \mathrm{~h}$ before being placed in a $30 \%$ Sucrose solution at $4{ }^{\circ} \mathrm{C}$ for $>24 \mathrm{~h}$. Following this cryo-protection step the brains were embedded in OCT using a peel-away mold and then stored at $-80^{\circ} \mathrm{C}$. Coronal $20 \mu \mathrm{m}$-think brain sections that contained the barrel cortex were cut and collected on-slide (wS1) using a cryostat (Leica, CM3050 S). The slides were then stored at $-80^{\circ} \mathrm{C}$ until further processing. For labeling thalamic input onto the tdTomato expressing cells an antibody staining was performed. For blocking, $0.75 \mathrm{uL}$ of PBS with $0.1 \%$ TritonX and $1.5 \%$ normal donkey serum was applied on the slide and left for $1 \mathrm{~h}$ at RT. Both primary and secondary antibodies were diluted in the same blocking solution. The primary, rabbit aVGlut2 (1:500, SySy 135 402), was left on the slide overnight $(>17 \mathrm{~h})$ at $4{ }^{\circ} \mathrm{C}$ while the secondary, donkey a Rabbit488 (1:1000, Thermo Fisher A21206), was left for $2 \mathrm{~h}$ at RT. The slides were then coverslipped using VectaShield Gold and stored at $4{ }^{\circ} \mathrm{C}$ for imaging.

\section{Confocal imaging and image processing of immunohistochemical samples.}

The slides were imaged using a Confocal Microscope (Olympus FV1000 with Fluoview software). All images were taken from L2/3 of the barrel cortex and of each of the processed brain 8-10 stacks were generated. To image the VGlut2 puncta a $60 \times$ oil-immersion objective was used and stacks were taken at $0.47-\mu \mathrm{m}$ distance.

A custom MATLAB script was used to analyze the VGlut2-tdTomato colocalization. The algorithm worked as follow: Image de-noising was performed using Wiener filtering followed by 2-D bilateral filtering. Wiener filter performs 2 $\mathrm{D}$ adaptive noise-removal resulting in a low pass filtered version of an intensity image that has been corrupted by stationary additive noise ${ }^{64}$ that is estimated based on statistics at a local neighborhood of each pixel. The bilateral filter is an edgepreserving nonlinear filter that smoothens a signal while preserving strong edges ${ }^{65}$ After de-noising, Z-stacks were binarized using multilevel Otsu thresholding ${ }^{66}$ for each $x y-, x z-$, and yz- slices separately, resulting in 3 binary 3D-stacks combined using logical AND operator (This very stringent binarisation operation makes sure the detected signal is noise free). Finally 3D binarized stacks for channel 1 (VGlut2) and 2 (VIP- or SST-tdTomato) were combined with another AND operator and 3D connected components analysis ${ }^{67}$ was used to count number of co-localizations. Number of co-localizations was normalized by total tdTomato signal for each image.

Viral injections. For these experiments the HTB reporter line was crossed with either VIPCre or SSTCre. The pups were injected with ASLV-A envelope glycoprotein (EnvA)-pseudotyped, glycoprotein-deleted rabies virus SADG-mCherry $(\text { EnvA })^{26}$ at either P4/P5 or P15. All pups were injected with $100 \mathrm{nl}$ of the virus, $120-170 \mu \mathrm{m}$ deep into the barrel field of the primary somatosensory cortex. The injections were done using a glass micropipette attached to a Nanolitre 2010 pressure injection apparatus (World Precision Instruments). After the surgeries, the animals were returned to their home-cage for 7 days to allow for adequate viral expression.

For the control rabies virus experiments VIPCre pups aged P15 were injected with $100 \mathrm{nl}$ of a 1:1 mix of pAAV-hSyn-FLEX-TVA-P2A-EGFP-2A-oG (gift from John Naughton, Addgene plasmid \# 85225) and rabies virus (see above). The injections were done at the same depth and using the same equipment as described above. The animals were sacrificed 7 days after the injection and all the brains were cleared as described below.

Tissue processing after viral injections. Seven days after the rabies virus injection the animals were transcardially perfused using $1 \mathrm{x}$ PBS and 4\% PFA. Following that, the brain were either processed for immunohistochemistry as described above or for tissue clearing. For immunohistochemistry the whole brain was cut at $20 \mu \mathrm{m}$ using a cryostat (Leica, CM3050 S) and collected on slide. Every third of the collected sections was stained with chicken- $\alpha$ GFP antibodies (abcam, ab13970, $1: 1000)$ to enhance the HTB signal.

The method used for hydrogel-based tissue clearing is explained in detail elsewhere ${ }^{68-70}$. The animals were transcardially perfused using $4 \%$ PFA and the brains post-fixed for $48 \mathrm{~h}$ in a Hydrogel solution (1\% PFA, 4\% Acrylamide, $0.05 \%$ Bis $)^{68,69}$ before the Hydrogel polymerization was induced at $37^{\circ} \mathrm{C}$. Following the polymerization the brains were immersed in $40 \mathrm{~mL}$ of $8 \%$ SDS and kept shaking at room temperature until the tissue was cleared sufficiently (10-40 days depending on the age of the animals). Finally, after $2-4$ washes in PBS, the brains were put into a self-made refractive index matching solution (RIMS ${ }^{71}$ for the last clearing step. They were left to equilibrate in $5 \mathrm{~mL}$ of RIMS for at least 4 days at RT before being imaged.

Imaging of rabies injected brains. A Slidescanner (Zeiss, AxioScan Z1) was used to image the stained sections. Mosaic images of the injected hemisphere were taken using a $20 \times$ objective. The obtained pictures were processed for analysis using the ZEN Software and Fiji.

After clearing, brains were attached to a small weight and loaded into a quartz cuvette, then submerged in RIMS and imaged using a home-built mesoscale selective plane illumination microscope (mesoSPIM) ${ }^{27}$. The microscope consists of a dual-sided excitation path using a fiber-coupled multiline laser combiner (405, $488,515,561,594,647 \mathrm{~nm}$, Omicron SOLE-6) and a detection path comprising an Olympus MVX-10 zoom macroscope with a $1 \times$ objective (Olympus MVPLAPO 1x), a filter wheel (Ludl 96A350), and a scientific CMOS (sCMOS) camera (Hamamatsu Orca Flash 4.0 V3). For imaging mCherry and eGFP a $594 \mathrm{~nm}$ excitation with a 594 long-pass filter (594 LP Edge Basic, AHF) and $488 \mathrm{~nm} \& 520 /$ 35 (BrightLine HC, AHF) were used respectively. The excitation paths also contain galvo scanners (GCM-2280-1500, Citizen Chiba) for light-sheet generation and reduction of streaking artifacts due to absorption of the light-sheet. In addition, the beam waist is scanning using electrically tunable lenses (ETL, Optotune EL-16-405D-TC-L) synchronized with the rolling shutter of the sCMOS camera. This axially scanned light-sheet mode (ASLM) leads to a uniform axial resolution across 
the field-of-view of 5-10 $\mu \mathrm{m}$ (depending on zoom and wavelength). Field of views ranged from $10.79 \mathrm{~mm}$ at $\times 1.25$ magnification (Pixel size: $5.27 \mu \mathrm{m}$ ) for overview datasets to $3.27 \mathrm{~mm}$ at $4 \times$ (Pixel size: $1.6 \mu \mathrm{m}$ ). Further technical details of the mesoSPIM are described elsewhere (www.mesospim.org). The images generated with the mesoSPIM were further processed using the Fiji and the Imaris software.

Analysis of cleared tissue imaging data. Close-up stacks taken from the barrel cortex of rabies injected and cleared SSTCre-HTB and VIPCre-HTB brains were initially processed through Fiji and z-stacked samples of superficial and deeper layers were saved after intensity thresholding. In each brain, barrels were segmented from layer 4 by drawing the vectors on barrels through scalar vector graphics' software (BoxySVG). Afterwards, automated neuron (starter- and presynaptic cells) detection was performed on maximum intensity projections of L $2 / 3$ green (HTB) and red (pre-synaptic cells) through a Deep Neural Network-based method, DeNeRD ${ }^{28}$. Initially, selected brain sections of high-resolution are segmented into smaller images by equally dividing the brain section into $100 \times$ 100 smaller sections, whereas each smaller image contains $\sim 20$ neurons. After annotating the neurons in a subset of these images (total $=160$ ), 2/3rd of them are randomly drawn for training and 1/3rd for testing purpose. A Simple Graphical User Interface (SiGUI) software, developed in MATLAB, is used to generate the ground-truth labels. Ground truth labels were generated by human experts who annotated the bounding boxes on top of the neurons. After generating the annotation, these brain images were fed to the DeNeRD for training the deep neural network (DNN). Four steps training procedure is applied that includes: (i) Training of the Region Proposal Network (RPN), (ii) Use the RPN from (i) to train a Fast RCNN, (iii) Retraining RPN by sharing weights with Fast RCNN of (ii). Finally, (iv) Retraining Fast RCNN using updated RPN. Epoch size of 500 is used with the initial learning rate of $1 \times 10^{-5}$ and $1 \times 10^{-6}$ in stages (i-ii) and (iii-iv), respectively. Training is performed by using NVIDIA Quadro P4000 GPU. The network is trained by minimizing the multi-task loss which corresponds to the each labeled Region of Interest, ROI (i.e. neuronal body) through the stochastic gradient descent algorithm. The mean average precision (F1 score) of 0.9 was achieved on the testing dataset. After the DNN is trained, each brain section from the barrel region is serially passed through the DNN and neuron segmentation is performed.

Then, the 2D Euclidean distance between each starter cell and each of the presynaptic cells present within a $800-\mu \mathrm{m}$ distance was measured using a custom MATLAB code. Plotted was the cumulative distribution of the distance for every starter cell as well as the average cumulative distribution of the percentage (probability) of pre-synaptic cells at a given Euclidian distance from the center of the starter cells.

Additional analysis were performed by randomizing the selection of starter and presynaptic cells in the barrel cortex images of each cleared brain. We started by taking the original number of starter cells and randomly selecting the number of presynaptic cells that matches the observed ratio between the two cell groups for each brain. Ten iterations were performed and the Euclidean distance between each starter and presynaptic cell was measured. Subsequently, the number of starter cells was decreased by one and the above process was repeated until only one starter cell was left. For each iteration, the cumulative distance distribution was calculated and plotted.

Statistical analysis. Data are represented as mean \pm s.e.m. unless stated otherwise. Statistical comparisons have been done using a paired Wilcoxon-signed rank test for paired data and two-tailed Mann-Whitney $U$ test for non-paired data. A Kolmogorov-Smirnov test was applied to statistically compare the cumulative distribution of the distance between starter- and pre-synaptic cells, and for decay tau comparisons. Two-tailed $t$-test was used to analyze the distance analysis of the glutamate uncaging evoked responses in L2/3. Significance threshold was set to $p<$ 0.05 ; in the figures, different degrees of evidence against the null hypothesis are indicated by asterisks $\left({ }^{*} p<0.05,{ }^{* *} p<0.01,{ }^{* *} p<0.001\right)$.

Reporting summary. Further information on research design is available in the Nature Research Reporting Summary linked to this article.

\section{Data availability}

Data are available upon request. Source data are provided with this paper.

\section{Code availability}

The DeNeRD code for neuron detection is available on Github (https://github.com/ itsasimiqbal/DeNeRD). Additional codes written for this paper are available upon request.

Received: 30 October 2019; Accepted: 12 October 2020; Published online: 12 November 2020

\section{References}

1. Ko, H. et al. The emergence of functional microcircuits in visual cortex. Nature 496, 96-100 (2013).

2. Van Der Loos, H. \& Woolsey, T. A. Somatosensory cortex: structural alterations following early injury to sense organs. Science 179, 395-398 (1973).

3. Killackey, H. P. Anatomical evidence for cortical subdivisions based on vertically discrete thalamic projections from the ventral posterior nucleus to cortical barrels in the rat. Brain Res. 51, 326-331 (1973).

4. Khazipov, R. et al. Early motor activity drives spindle bursts in the developing somatosensory cortex. Nature 432, 758-761 (2004).

5. Akhmetshina, D., Nasretdinov, A., Zakharov, A., Valeeva, G. \& Khazipov, R. The nature of the sensory input to the neonatal rat barrel cortex. J. Neurosci. 36, 9922-9932 (2016).

6. Arakawa, H. \& Erzurumlu, R. S. Role of whiskers in sensorimotor development of C57BL/6 mice. Behav. Brain Res. 287, 146-155 (2015).

7. Landers, M. \& Philip Zeigler, H. Development of rodent whisking: Trigeminal input and central pattern generation. Somatosens. Mot. Res. 23, 1-10 (2006).

8. Welker, W. I. Analysis of Sniffing of the Albino Rat 1. Behaviour. 22, 223-244 (1964)

9. Lee, S., Kruglikov, I., Huang, Z. J., Fishell, G. \& Rudy, B. A disinhibitory circuit mediates motor integration in the somatosensory cortex. Nat. Neurosci. 16, $1662-1670$ (2013).

10. Muñoz, W., Tremblay, R., Levenstein, D. \& Rudy, B. Layer-specific modulation of neocortical dendritic inhibition during active wakefulness. Science 355, 954-959 (2017).

11. Gentet, L. J. et al. Unique functional properties of somatostatin-expressing GABAergic neurons in mouse barrel cortex. Nat. Neurosci. 15, 607-612 (2012).

12. Pfeffer, C. K., Xue, M., He, M., Huang, Z. J. \& Scanziani, M. Inhibition of inhibition in visual cortex: the logic of connections between molecularly distinct interneurons. Nat. Neurosci. 16, 1068-1076 (2013).

13. $\mathrm{Pi}, \mathrm{H}$.-J. J. et al. Cortical interneurons that specialize in disinhibitory control. Nature 503, 521-4 (2013).

14. Karnani, M. M. et al. Opening holes in the blanket of inhibition: localized lateral disinhibition by VIP interneurons. J. Neurosci. 36, 3471-3480 (2016).

15. Pinto, L. \& Dan, Y. Cell-type-specific activity in prefrontal cortex during goaldirected behavior. Neuron 87, 437-451 (2015).

16. Fu, Y. et al. A cortical circuit for gain control by behavioral state. Cell 156, 1139-1152 (2014).

17. Sachidhanandam, S., Sermet, B. S. \& Petersen, C. C. H. Parvalbuminexpressing GABAergic neurons in mouse barrel cortex contribute to gating a goal-directed sensorimotor transformation. Cell Rep. 15, 700-706 (2016).

18. Audette, N. J., Urban-Ciecko, J., Matsushita, M. \& Barth, A. L. POm thalamocortical input drives layer-specific microcircuits in somatosensory cortex. Cereb. Cortex 28, 1312-1328 (2018).

19. Williams, L. E. \& Holtmaat, A. Higher-order thalamocortical inputs gate synaptic long-term potentiation via disinhibition. Neuron. https://doi.org/ 10.1016/j.neuron.2018.10.049 (2018)

20. Yu, J., Hu, H., Agmon, A. \& Svoboda, K. Recruitment of GABAergic interneurons in the barrel cortex during active tactile behavior. Neuron. https://doi.org/10.1016/j.neuron.2019.07.027 (2019)

21. van der Bourg, A. et al. Layer-specific refinement of sensory coding in developing mouse barrel cortex. Cereb. Cortex 27, 4835-4850 (2017).

22. Golshani, P. et al. Internally mediated developmental desynchronization of neocortical network activity. J. Neurosci. 29, 10890-10899 (2009).

23. Che, A. et al. Layer I interneurons sharpen sensory maps during neonatal development. Neuron 99, 98-116.e7 (2018).

24. Ikezoe, K., Tamura, H., Kimura, F. \& Fujita, I. Decorrelation of sensoryevoked neuronal responses in rat barrel cortex during postnatal development. Neurosci. Res. 73, 312-320 (2012).

25. Karnani, M. M. M. et al. Cooperative Subnetworks of molecularly similar interneurons in mouse neocortex. Neuron 90, 86-100 (2016).

26. Wickersham, I. R. et al. Monosynaptic restriction of transsynaptic tracing from single, genetically targeted. Neurons Neuron 53, 639-647 (2007).

27. Voigt, F. F. et al. The mesoSPIM initiative: open-source light-sheet microscopes for imaging cleared tissue. Nat. Methods. https://doi.org/10.1038/ s41592-019-0554-0 (2019)

28. Iqbal, A., Sheikh, A. \& Karayannis, T. DeNeRD: high-throughput detection of neurons for brain-wide analysis with deep learning. Sci. Rep. 9, 1-13 (2019).

29. Adesnik, H., Bruns, W., Taniguchi, H., Huang, Z. J. \& Scanziani, M. A neural circuit for spatial summation in visual cortex. Nature 490, 226-230 (2012).

30. Xu, X. \& Callaway, E. M. Laminar specificity of functional input to distinct types of inhibitory cortical neurons. J. Neurosci. 29, 70-85 (2009).

31. Zhang, W. \& Bruno, R. M. High-order thalamic inputs to primary somatosensory cortex are stronger and longer lasting than cortical inputs. Elife 8, 1-22 (2019). 
32. Sermet, B. S. et al. Pathway-, layer-and cell-type-specific thalamic input to mouse barrel cortex. Elife 8, 1-28 (2019).

33. Ciabatti, E., González-Rueda, A., Mariotti, L., Morgese, F. \& Tripodi, M. Lifelong genetic and functional access to neural circuits using self-inactivating rabies virus. Cell 170, 382-392.e14 (2017).

34. Kim, E. J., Jacobs, M. W., Ito-Cole, T. \& Callaway, E. M. Improved monosynaptic neural circuit tracing using engineered rabies virus glycoproteins. Cell Rep. 15, 692-699 (2016).

35. Reardon, T. R. et al. Rabies virus CVS-N2c $\delta G$ strain enhances retrograde synaptic transfer and neuronal viability. Neuron 89, 711-724 (2016).

36. Tuncdemir, S. N. et al. Early somatostatin interneuron connectivity mediates the maturation of deep layer cortical circuits. Neuron 89, 521-535 (2016).

37. Deschênes, M., Veinante, P. \& Zhang, Z. W. The organization of corticothalamic projections: reciprocity versus parity. Brain Res. Rev. 28, 286-308 (1998).

38. Groh, A., de Kock, C. P. J., Wimmer, V. C., Sakmann, B. \& Kuner, T. Driver or coincidence detector: modal switch of a corticothalamic giant synapse controlled by spontaneous activity and short-term depression. J. Neurosci. 28, 9652-9663 (2008).

39. Jiang, X. et al. Principles of connectivity among morphologically defined cell types in adult neocortex. Science 350, aac9462 (2015).

40. Wall, N. R. et al. Brain-wide maps of synaptic input to cortical interneurons. J. Neurosci. 36, 4000-4009 (2016).

41. Arbuthnott, G. W., MacLeod, N. K., Maxwell, D. J. \& Wright, A. K. Distribution and synaptic contacts of the cortical terminals arising from neurons in the rat ventromedial thalamic nucleus. Neuroscience 38, 47-60 (1990).

42. Collins, D. P., Anastasiades, P. G., Marlin, J. J. \& Carter, A. G. Reciprocal circuits linking the prefrontal cortex with dorsal and ventral thalamic nuclei. Neuron 98, 366-379.e4 (2018)

43. Chevalier, G. \& Deniau, J. M. Disinhibition as a basic process in the expression of striatal functions. II. The striato-nigral influence on thalamocortical cells of the ventromedial thalamic nucleus. Brain Res. 334, 227-233 (1985).

44. Jones, E. G. \& Leavitt, R. Y. Retrograde axonal transport and the demonstration of non-specific projections to the cerebral cortex and striatum from thalamic intralaminar nuclei in the rat, cat and monkey. J. Comp. Neurol. 154, 349-377 (1974).

45. Cruikshank, S. J. et al. Thalamic control of layer 1 circuits in prefrontal cortex. J. Neurosci. 32, 17813-17823 (2012).

46. Herkenham, M. The afferent and efferent connections of the ventromedial thalamic nucleus in the rat. J. Comp. Neurol. 183, 487-517 (1979).

47. Kuramoto, E. et al. Two types of thalamocortical projections from the motor thalamic nuclei of the rat: a single neuron-tracing study using viral vectors. Cereb. Cortex 19, 2065-2077 (2009).

48. Ma, Y., Hu, H., Berrebi, A. S., Mathers, P. H. \& Agmon, A. Distinct subtypes of somatostatin-containing neocortical interneurons revealed in transgenic mice. J. Neurosci. 26, 5069-5082 (2006).

49. Sych, Y., Chernysheva, M., Sumanovski, L. T. \& Helmchen, F. High-density multi-fiber photometry for studying large-scale brain circuit dynamics. bioRxiv 16, 422857 (2018).

50. Taniguchi, H. et al. A resource of cre driver lines for genetic targeting of GABAergic. Neurons Cereb. Cortex. Neuron 71, 995-1013 (2011).

51. Madisen, L. et al. A robust and high-throughput Cre reporting and characterization system for the whole mouse brain. Nat. Neurosci. 13, 133-140 (2010).

52. Li, Y. et al. Molecular layer perforant path-associated cells contribute to feedforward inhibition in the adult dentate gyrus. Proc. Natl Acad. Sci. USA 110, 9106-11 (2013).

53. Stosiek, C., Garaschuk, O., Holthoff, K. \& Konnerth, A. In vivo two-photon calcium imaging of neuronal networks. Proc. Natl Acad. Sci. USA 100, 7319-7324 (2003).

54. Pologruto, T. A., Sabatini, B. L. \& Svoboda, K. ScanImage: flexible software for operating laser scanning microscopes. Biomed. Eng. Online 2, 13 (2003).

55. Guizar-Sicairos, M., Thurman, S. T. \& Fienup, J. R. Efficient subpixel image registration algorithms. Opt. Lett. 33, 156 (2008).

56. Peron, S., Chen, T. W. \& Svoboda, K. Comprehensive imaging of cortical networks. Curr. Opin. Neurobiol. 32, 115-123 (2015)

57. Kerlin, A. M., Andermann, M. L., Berezovskii, V. K. \& Reid, R. C. Broadly tuned response properties of diverse inhibitory neuron subtypes in mouse visual cortex. Neuron 67, 858-871 (2010).

58. Khan, A. G. et al. Distinct learning-induced changes in stimulus selectivity and interactions of GABAergic interneuron classes in visual cortex. Nat. Neurosci. 21, 851-859 (2018).

59. Miyoshi, G. et al. Genetic fate mapping reveals that the caudal ganglionic eminence produces a large and diverse population of superficial cortical interneurons. J. Neurosci. 30, 1582-1594 (2010).
60. Kawaguchi, Y. Physiological subgroups of nonpyramidal cells with specific morphological characteristics in layer II/III of rat frontal cortex. J. Neurosci. 15, 2638-55 (1995).

61. Shepherd, G. M. G., Pologruto, T. A. \& Svoboda, K. Circuit analysis of experience-dependent plasticity in the developing rat barrel cortex. Neuron $\mathbf{3 8}$, 277-289 (2003).

62. Anastasiades, P. G. et al. GABAergic interneurons form transient layerspecific circuits in early postnatal neocortex. Nat. Commun. 7, 10584 (2016).

63. Callaway, E. M. \& Katz, L. C. Photostimulation using caged glutamate reveals functional circuitry in living brain slices. Proc. Natl Acad. Sci. USA 90, 7661-7665 (1993).

64. Lim, J. S. Two-Dimensional Signal and Image Processing (Prentice-Hall, Inc. Upper Saddle River, 1990).

65. Tomasi, C. \& Manduchi, R. Bilateral filtering for gray and color images. Sixth Int. Conf. Comput. Vis. https://doi.org/10.1109/ICCV.1998.710815 (1998).

66. Otsu, N. A threshold selection method from gray-level histograms. IEEE Trans. Syst. Man. Cybern. 9, 62-66 (1979).

67. Ronse, C. \& Devijver, P. A. Connected components in binary images: the detection problem (John Wiley \& Sons, Inc, New York, 1984).

68. Chung, K. et al. Structural and molecular interrogation of intact biological systems. Nature 497, 332-7 (2013).

69. Ye, L. et al. Wiring and molecular features of prefrontal ensembles representing distinct experiences. Cell 165, 1776-1788 (2016).

70. Tomer, R., Ye, L., Hsueh, B. \& Deisseroth, K. Advanced CLARITY for rapid and high-resolution imaging of intact tissues. Nat. Protoc. 9, 1682-97 (2014)

71. Yang, B. et al. Single-cell phenotyping within transparent intact tissue through whole-body clearing. Cell 158, 945-958 (2014).

\section{Acknowledgements}

We thank O. Hanley for the production of the pseudotyped rabies virus used in this study, A. Engmann and the Salk Institute for providing the cell lines for this production, P. Bethge for his help with the $2 \mathrm{P}$ setup, $\mathrm{H}$. Kasper and M. Wieckhorst for technical assistance and O. Weinmann for his advice on immunostainings. We would also like to thank L. Egolf for her assistance with the VIPCre, SSTCre and Ail4 mouse lines. Slidescanner imaging and data analysis was performed with equipment maintained by the Center for Microscopy and Image Analysis (ZMB), University of Zurich. We thank C. Aemisegger at the ZMB for her assistance with the Slidescanner. This work was supported by grants from the European Research Council (ERC, 679175, T.K and 670757, F.H), the Swiss National Science Foundation (SNSF, 31003A_170037, T.K.), Fond zur Förderung des Akademischen Nachwuchs of the UZH Alumni (T.K.) and the Swiss Foundation for Excellence in Biomedical Research (R.K. and T.K.).

\section{Author contributions}

R.K. performed the anatomical mapping, tissue clearing, immunohistochemistry and wrote the manuscript; R.V. performed functional electrophysiology experiments, in vitro $\mathrm{Ca}^{2+}$ imaging, contributed to the analysis of in vitro electrophysiological data and wrote the paper; A.v.d.B. performed in vivo $\mathrm{Ca}^{2+}$ imaging experiments and initial virus injections; A.Ö.A. performed the analysis of the in vivo $\mathrm{Ca}^{2+}$ imaging data, puncta colocalization in histology, analysis of the in vitro electrophysiology data and decoder analysis; A.I. developed the deep neural network-based neuron detector pipeline (DeNeRD), did the analysis of the rabies-traced cells and the their distribution in the cortex. F.F.V. built, provided access to and guidance for the light-sheet microscope, F.H. provided access to the Light-Sheet Microscope and a two-photon microscope. D.K. and A.A. provided initial guidance on tissue clearing. T.K. conceptualized the study, designed the experiments and wrote the paper.

\section{Competing interests}

The authors declare no competing interests.

\section{Additional information}

Supplementary information is available for this paper at https://doi.org/10.1038/s41467 020-19427-z.

Correspondence and requests for materials should be addressed to T.K.

Peer review information Nature Communications thanks Josef Bischofberger, Lisa Topolnik, and other, anonymous, reviewers for their contributions to the peer review of this work.

Reprints and permission information is available at http://www.nature.com/reprints

Publisher's note Springer Nature remains neutral with regard to jurisdictional claims in published maps and institutional affiliations. 
(c) (i) Open Access This article is licensed under a Creative Commons Attribution 4.0 International License, which permits use, sharing, adaptation, distribution and reproduction in any medium or format, as long as you give appropriate credit to the original author(s) and the source, provide a link to the Creative Commons license, and indicate if changes were made. The images or other third party material in this article are included in the article's Creative Commons license, unless indicated otherwise in a credit line to the material. If material is not included in the article's Creative Commons license and your intended use is not permitted by statutory regulation or exceeds the permitted use, you will need to obtain permission directly from the copyright holder. To view a copy of this license, visit http://creativecommons.org/ licenses/by/4.0/.

(C) The Author(s) 2020 